\title{
ANALYSIS OF WOVEN FABRIC STRENGTHS: PREDICTION OF FABRIC STRENGTH UNDER UNIAXIAL AND BIAXIAL EXTENSIONS
}

\author{
Ning Pan \\ Division of Textiles and Clothing, Biological and Agricultural Engineering Department, University of California, Davis, \\ California 95616, USA
}

\begin{abstract}
This study of woven fabric strength begins by focusing on the effects of the crossing points, where warp and weft yarns interlace with each other to form a fabric, towards the ultimate fabric tensile strength. When a fabric is under uniaxial or biaxial tension, the yarn-yarn interactions at the crossing points are found to consist of two components, a pressure-independent adhesive component and a pressure-dependent frictional one, of which the latter is proved to be dominant. On the basis of the analysis, the yarn critical length in the fragmentation process during fabric extension is defined and calculated. Moreover, by treating the fabric as an assemblage of yarn bundles, the distribution density function of the strengths between individual yarns in the same bundle is provided based on the single yarn strength distribution, and the fracture process of the bundle can be better described using this function.
\end{abstract}

Then, by incorporating the critical yarn length into the distribution density function of the yarn strength, the in situ behavior of the yarn bundle in a fabric is predicted. Following the chain of sub-bundle model, the mean fabric strength and the fabric stress/strain curve are predicted more realistically for both uniaxial and biaxial tension cases.

The predictions of the present theory compare favorably with the measured fabric strengths both previously reported and tested in the present work. A detailed parametric study is also carried out.

Keywords: woven fabric strength, uniaxial and biaxial extensions

\section{INTRODUCTION}

Although biaxial woven fabrics existed long before the Industrial Revolution, and fabric manufacturing techniques have since advanced enormously, our understanding of the mechanical behavior of the fabrics is still very limited.

In the past few decades, instead of simply being used as a clothing material, woven fabric has gained wider applications in many other areas including some load-carrying structural utilizations. Recently, woven fabrics have been applied as the fiber preform for fiber-reinforced composites. Consequently, it becomes increasingly desirable to develop a more advanced mechanistic theory governing the fabric responses under various loading situations so as to provide a powerful design tool for fabric structures in heavy load-carrying applications.

So far, attempts to investigate the mechanical behavior of woven fabrics have more or less followed three paths. The well-known Peirce geometrical mode ${ }^{1}$ has been considered to be the first such undertaking. By assuming a woven fabric as a highly idealized geometrical object, Peirce was able to describe the deformational behavior of the fabric under external loading. Painter ${ }^{2}$ and Love $^{3}$ further refined this technique in their analyses of fabric properties. Yet, because of the level of ignorance of the mechanistic load/deformation relationships and excessively idealized features of the constituent yarns in the fabric, this technique is very limited in terms of reliability and applicability.

By incorporating the mechanisms of force equilibrium into the geometrical model and lifting some of the over-restrained assumptions, the second analytical approach was developed. For example, Hearle et al. ${ }^{4}$ carried out extensive studies on the mechanical behavior of woven fabrics by applying both geometrical and mechanistic analysis. Taylor ${ }^{5}$ used a mechanical model based loosely on Peirce's fabric geometry to examine the tear strength of a fabric as a function of the yarn strength and the force required to slip one yarn set over another. Postle et al. ${ }^{6}$ applied the energy method to determine yarn and fabric deformation by minimizing the strain-energy function. However, the problem associated with this technique is the complicated, often cumbersome, mathematical equations. Further study of fabric tear strength has recently been reported by Scelzo et al.$^{7-8}$

Another approach is based on continuum mechanics by treating the fabric as a continuum laminate. Kilby ${ }^{9}$ was perhaps among the first to apply this approach to study the anisotropic nature of woven fabrics, i.e. the directional dependence of the tensile modulus. Also, 
by treating the fabric as a spatially periodic interlaced network of orthogonal yarns and by modeling the individual yarns as extensible elastica, Warren ${ }^{10}$ was able to determine theoretically the in-plane linear elastic constants of a woven fabric. The drawback of this type of analysis lies in the neglect of the structural details and the yarn-yarn interactions in the fabric. This technique therefore is mainly applicable to the prediction of the elastic material constants rather than the strength of a fabric.

A new stimulation to the study of the mechanics of fabrics has emerged since the 1980s from researchers in the composites field. Fabrics and other textile structures as preform for the so-called textile structural composites have attracted more and more attention from the composites community. This stems from the need for improvements in intra- and interlamiar strength and damage tolerance, especially in thick-section composites. Textile composites offer the potential of providing adequate structural integrity as well as shapeability for near-net-shape manufacturing. The major theoretical contributors in this area seem to include mainly Chou and his colleagues, and their work is best summarized by Chou, ${ }^{11}$, Ko and Pastore, ${ }^{12}$ Pastore and Gowayed, ${ }^{13}$ Hearle and $\mathrm{Du},{ }^{14}$ and $\mathrm{Li}$ et al. ${ }^{15}$ Largely, the technique in analysing woven fabrics of $\mathrm{Chou}^{11}$ is also based on the laminate theory of continuum mechanics. By treating a fabric as a laminate with or without inclusion of the yarn undulation (crimping) effect, it was possible to establish the constitutive equations for various fabrics. Again, this method only leads to the prediction of the elastic material constants as well as fabric thermal properties, and is unable to tackle the problem of fabric strengths which rely primarily on the property extremes in the system. Additionally, in all the analysis on woven fabric-reinforced composites, the yarn-yarn interactions at the fabric interlacing points have been ignored. As is demonstrated later in the present study, the yarn-yarn interaction at the fabric crossing points provides a fundamental mechanism of fabric strength enhancement, and it will likely play an important role in reinforcing the composite.

Shahpurwala and Schwartz ${ }^{16}$ attempted to predict the tensile strength of woven fabrics using derived strength distributions of the constituent yarns. By treating the fabric as a bundle of yarns, they applied the statistical theory of Daniels ${ }^{17}$ to predict the strength of the fabric. They found that when the interactions between yarns are ignored, the theoretical prediction is as low as a factor of 0.67 . They concluded that a more realistic prediction can be obtained if the fabric is modeled as a bundle of yarn segment whose length, termed as effective or critical length, is much shorter than the original yarn length. By back calculation using the weakest-link scaling based on the known fabric and yarn strength distributions, they determined the critical lengths ranging from a high of $18.7 \mathrm{~mm}$ to a low of $6 \mathrm{~mm}$, depending on the type of fabrics. Their study proves that the mechanical behavior of a yarn in a fabric differs considerably from the observed ex situ yarn performance. However, this study fails to establish a theoretical relationship between the critical subbundle yarn length and the interactions between yarns in a tensioned fabric. Instead, they proceeded with a more or less empirical approach. A similar approach has been adopted by Boyce et al. ${ }^{18}$ and Seo et al. ${ }^{19}$ to more accurately predict the fabric tensile strength.

There have also been studies focusing on the mechanical behavior of fabrics under biaxial loading, for instance, as reported by Clulow and Taylor, ${ }^{20}$ Freeston et al., ${ }^{21}$, Reichardt et al..$^{22}$ and Skelton and Freeston. ${ }^{23}$ These studies, however, mainly dealt with the establishment of the constitutive relationships of fabrics specifying the elastic performance, and rarely touched the issue of strength prediction.

Recently Pan and Yoon ${ }^{24}$ have proposed an analytical model to describe the yarn pullout process from a woven fabric. This theory is able to predict the relationship of the maximum pullout load and the embedded yarn length in a woven fabric. The maximum load is shown in the theory to be related to the yarn-yarn interactions, and the mechanical and geometrical properties of the fabric and the yarn. This approach is useful in understanding the nature of the yarn interactions in a fabric and the structurereinforcing mechanism of woven fabrics.

The present work is aimed to develop a theoretical approach for fabric strength prediction, based on the results achieved by previous studies. The basic principle in this work is quite similar to the model proposed by Shahpurwala and Schwartz ${ }^{16}$ that a fabric be treated as a system of chains formed by yarn sub-bundles whose length is equal to the so-called critical length. One of the major features in the present study is the derivation of the expression for the critical yarn length, $l_{c}$, based on the yarn-yarn interactions and the mechanical and geometrical properties of the fabric and its constitucnt yarns. The yarn-yarn interactions are characterized in terms of the adhesive and frictional actions occurring at the interlacing points where the yarns in warp and weft systems contact and interact. By incorporating the adhesive and frictional mechanisms into the analysis, we will be able to include the transverse extension which enhances the frictional force, and the yarn surface topology, which affects both the adhesive and the frictional actions, into our analysis. Consequently we are able to predict the fabric strength and its variation at both uniaxial and biaxial extension cases.

It becomes known that a fabric is an extremely complex structure for mechanistic analysis. There exist several structural levels from fibers to yarns and 
eventually to the fabric. Each level has its own geometrical and mechanical variables which control or influence to varying degrees the fabric behavior. It will be a formidable or even impossible task to include all these variables into a fabric model without simplification. This simplification is done in the present work in three ways. First, we approximate a real fabric structure with an idealized model of more regular geometry; second, we exclude in our model the factors with ignorable or only marginal effects on the final results; last, for the variables included in our model, we express those unmeasurable variables in terms of the measurable ones. Through these approaches, we can retained the essence of the physics but avoid unnecessary complexity so as to make our model concise, more user-friendly, and easy to apply.

To begin with, we adopt the following assumptions in our analysis:

1. The tensile strength distribution of the individual yarns is of Weibull form.

2. The yarn jamming effect during fabric extension is negligible. Evidently, this assumption is more acceptable when dealing with a fabric with tight structure. Moreover, in a biaxial loading case where extension is exerted on both warp and weft directions, mutually perpendicular to each other, the yarn jamming effect during fabric stretch is less severe than in a uniaxial loading case.

3. Variations, and their changes during fabric extension, of the structural and geometrical parameters of a fabric are negligible.

4. The interactions between yarns in a fabric under extension will not affect the form of the strength distribution function of the individual yarns.

5. When a yarn in a fabric breaks, the load it was carrying is equally shared among the surviving yarns. The effects of stress concentration and dynamic wave propagation are ignored.

\section{FABRIC TENSILE STRENGTH AS A STOCHASTIC VARIABLE}

A biaxial woven fabric can be treated as an assembly of two systems of yarn bundles perpendicularly interlaced with each other in a two-dimensional format. Therefore, fabric strength is the resultant of the yarn strength taking into account the interactions between the two yarn systems.

\subsection{Weibull statistics of the strength of a single yarn}

According to the above assumption of the Weibull distribution function of yarn tensile strength, for a single yarn with length $l_{y}$, the probability of its breaking load being $\sigma_{\mathrm{y}}$ can be described by a two-parameter Weibull function:

$$
F\left(\sigma_{\mathrm{y}}\right)=1-\exp \left[-l_{\mathrm{y}} \alpha_{\mathrm{y}} \sigma_{\mathrm{y}}^{\beta}\right]
$$

where $\alpha_{\mathrm{y}}$ is the Weibull scale parameter, and $\beta_{\mathrm{y}}$ is the shape parameter of the yarn. The shape parameter is an indicator of the variation in yarn breaking loads. A higher $\beta_{\mathrm{y}}$ value corresponds to a lower variation, and when $\beta_{\mathrm{y}} \rightarrow \infty$, the variation would approach zero and the yarn breaking load would be independent of its length.

The mean or the expected value of the yarn breaking load, $\overline{\sigma_{y}}$ can then be calculated as:

$$
\overline{\sigma_{y}}=\left(l_{\mathrm{y}} \alpha_{\mathrm{y}}\right)^{-\left(1 / \beta_{\mathrm{y}}\right)} \Gamma\left(1+\frac{1}{\beta_{\mathrm{y}}}\right)
$$

where $\Gamma$ is the Gamma function, and the standard deviation of the breaking load is given by:

$$
\Theta_{\mathrm{y}}=\overline{\sigma_{\mathrm{y}}}\left(\frac{\Gamma\left(1+\frac{2}{\beta_{\mathrm{y}}}\right)}{\Gamma^{2}\left(1+\frac{1}{\beta_{\mathrm{y}}}\right)}-1\right)^{\frac{1}{2}}
$$

It should be noted here that we have established these relationships between the yarn tensile strength and its Weibull parameters in a generalized manner. The effects on yarn strength due to factors such as the fiber end slippage in a staple yarn, fiber migration, and the fiber obliquity have all been reflected in the values of the Weibull parameters. However, it has been shown recently by Realff $e t a l .^{25}$ that there has been a demonstrated failure mechanism change for short fiber yarns, reflected by the change of the value of the Weibull shape parameter, $\beta_{y}$, as a function of the gauge length at which the yarn is tested. Since we will use in our model the Weibull shape parameter tested at the standard gauge length, the effect of this observation by Realff $e t$ al. will be evaluated in the specific examples presented towards the end of the paper.

\subsection{The fabric strength excluding the effects of yarn-yarn interactions}

The tensile behavior of a fabric would be identical to that of its constituent yarns if all yarns were uniform in their tensile propertics, and if the interactions between the two perpendicular yarn systems were negligible. Unfortunately, in reality, these two factors are too significant to be excluded; this complicates the otherwise very straightforward relationship.

Let us first consider only one yarn bundle system where $N_{\mathrm{y}}$ yarns form a parallel bundle with no contact or interaction between the individual yarns. As there is more or less variation between the mechanical behavior of the individual yarns in the system, this will inevitably lead to a discrepancy between the properties of the single yarns and of the yarn bundle. Because of the statistical nature of single yarn 
strength, the yarn bundle strength will obey a statistical distribution as well. Based on the analysis by Daniels, ${ }^{17}$ for a large bundle of high $N_{\mathrm{y}}$ value, the density distribution function of the bundle strength $\sigma_{\mathrm{b}}$ approaches a normal form:

$$
H\left(\sigma_{\mathrm{b}}\right)=\frac{1}{\sqrt{2 \pi} \Theta_{\mathrm{b}}} \exp \left[-\frac{\left(\sigma_{\mathrm{b}}-\overline{\sigma_{\mathrm{b}}}\right)^{2}}{2 \Theta_{\mathrm{b}}^{2}}\right]
$$

where $\overline{\sigma_{\mathrm{b}}}$ is the expected value of the bundle strength and can be calculated from the yarn parameters as:

$$
\overline{\sigma_{\mathrm{b}}}=\left(l_{\mathrm{y}} \alpha_{\mathrm{y}} \beta_{\mathrm{y}}\right)^{-\frac{1}{\beta_{\mathrm{y}}}} \exp \left(-\frac{1}{\beta_{\mathrm{y}}}\right)
$$

and $\Theta_{b}$ is the standard deviation of the strength:

$$
\Theta_{\mathrm{b}}^{2}=\left(l_{\mathrm{y}} \alpha_{\mathrm{y}} \beta_{\mathrm{y}}\right)^{-\frac{2}{\beta_{\mathrm{y}}}}\left[\exp \left(-\frac{1}{\beta_{\mathrm{y}}}\right)\right]\left[1-\exp \left(-\frac{1}{\beta_{\mathrm{y}}}\right)\right] N_{y}^{-1}
$$

As is well known, the strength of this yarn bundle is lower than that of its constituent yarns. This can be seen by comparing eqns (2) and (5). The strength variation of the yarn bundle is also smaller (depending among other factors on its size $N_{\mathrm{y}}$ ) than that of the single yarn given in eqn (3).

It is apparent that the tensile modulus of this yarn bundle is taken here to be identical to that of the yarn.

To conclude, the strength variation between individual yarns will result in a reduction of both mean strength and the strength variation of the yarn bundle formed. Furthermore, if the interactions between yarns in the fabric are ignored, a fabric can be treated as a yarn bundle system in either of the two directions, i.e. the longitudinal (the warp) or the transverse (the weft) direction, and then eqn (5) in fact gives the fabric mean strength per yarn. So, according to the above theory, the fabric mean strength per yarn, as well as its standard deviation, will be lower than that of its constituent yarns, or the fabric is weaker than the yarns owing to the property variation between the yarns. This conclusion is actually in contradiction with the experimental results which have shown that the mean fabric strength per yarn is generally greater than the mean strength of its constituent yarns. Obviously, the main cause for this discrepancy lies in the exclusion of the effects of the yarn-yarn interactions in the above analysis. As shown below, these interactions will reinforce the fabric structure so as to achieve a fabric strength higher than that of the yarns. As the yarn-yarn interactions are determined by many statistical variables, this leads to a fabric strength of probabilistic or stochastic nature.

From now on, unless specified otherwise, all the analyses will be focused on the warp direction in a fabric.

\subsection{Yarn-yarn interactions at interlacing points, the critical length and the in situ yarn strength}

In order to include the yarn-yarn interactions in our model, we have to take into consideration the second yarn bundle system, which is in the direction perpendicular to and is interlaced with the above yarn bundle system to form a self-locked planar fibrous system-a woven fabric.

The interlacing (or crossing) points are the major locations where interactions between yarns in the two bundle systems take place, through which the yarns form an interlocked structure. Without the interactions occurring at the interlacing points, a woven fabric would be equivalent to a system of two sheets, each made of parallel but isolated yarns; the resultant properties would be entirely different from those of a practical fabric. In other words, the yarn interaction at the crossing points is the essential feature for a woven fabric, and will affect more or less all the fabric properties. Therefore, these interactions can be used as an indicator of, or a probe to, the various fabric properties, and, according to Pan and Yoon, ${ }^{24}$ can be investigated through a single yarn pullout test from the fabric.

As revealed by the statistical chain of sub-bundle theory first applied to woven fabric by Shahpurwala and Schwartz, ${ }^{16}$ it is expected that yarns, once woven into a fabric system, will behave differently due to the interactions between yarns in the fabric under cxtension, and the interactions will inevitably alter the properties of the yarns. Because of these interactions, the fabric is enhanced, and the concept of chain of sub-bundle theory can better specify this enhancement effect. Based on the theory, a fabric can be considered equivalent to a system made of chains of sub-bundles of yarns whose length is defined by a critical length $l_{\text {c }}$. We are going to derive a theoretical expression for $l_{\mathrm{c}}$ in terms of the fabric and yarn properties.

As stated above, the yarn-yarn interactions in a woven fabric mainly take place at the yarn crossing points through yarn-yarn contact. However, the contact area at an interlacing point between a warp and a weft yarn is only partial, as shown in Fig. 1 of an idealized illustration of a plain weave. If an external tension $\sigma_{\mathrm{y}}$ is applied to the fabric, a force equilibrium can be established at a differential portion $\mathrm{d} x$ as:

$$
\frac{L_{\mathrm{y}}}{2} \tau_{\mathrm{y}} n_{\mathrm{y}} \mathrm{d} x=\mathrm{d} \sigma_{\mathrm{y}}
$$

where, as illustrated in Fig. 1(a), $t_{\mathrm{y}}$ and $w_{\mathrm{y}}$ are the shorter and longer axes of the yarn cross section, $\tau_{\mathrm{y}}$ is the shear resistance, and $L_{y} / 2$ is the actual contact length between yarns, for one contact point. Here $L_{y}$ is the circumference of the yarn cross section, i.e.

$$
L_{\mathrm{y}}=1 \cdot 51 \pi\left(t_{\mathrm{y}}+w_{\mathrm{y}}\right)-\sqrt{\left(t_{\mathrm{y}} w_{\mathrm{y}}\right)}
$$




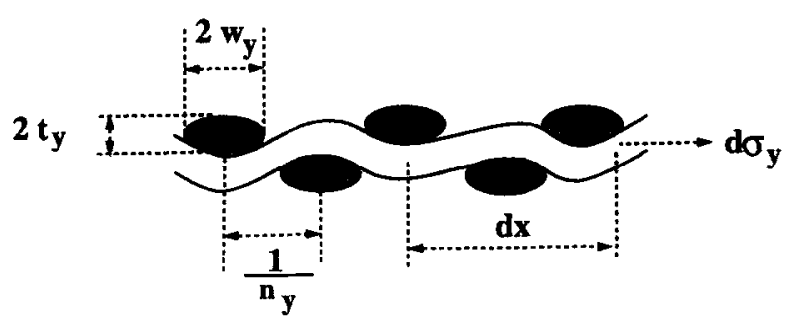

(a )

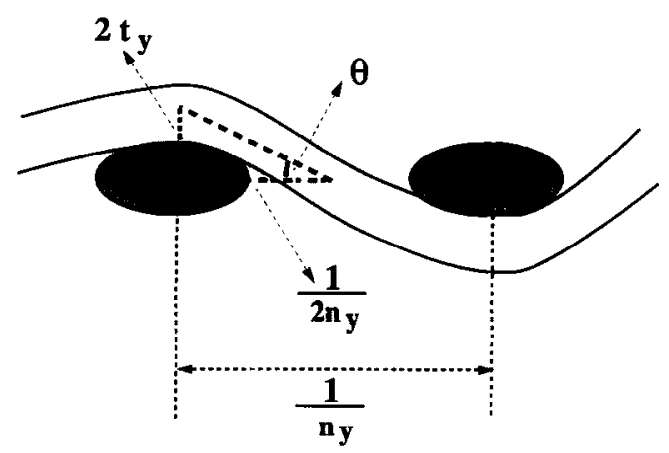

(b)

Fig. 1. Idealized geometrical features at yarn interlacing points of a plain weave fabric: (a) the dimensions of the yarn cross-section and spacing; (b) the interlacing angle.

and $n_{y}$ is the fabric count (number of yarns per fabric length).

If $\sigma_{y}$ is increased to the current in situ tensile breaking load of the yarn, it follows that the yarn critical length $l_{c}$ is given as:

$$
\frac{L_{\mathrm{y}}}{2} \tau_{\mathrm{y}} n_{\mathrm{y}} l_{\mathrm{c}}=\sigma_{\mathrm{y}}
$$

or

$$
l_{\mathrm{c}}=\frac{2}{L_{\mathrm{y}} n_{\mathrm{y}}} \frac{\sigma_{\mathrm{y}}}{\tau_{\mathrm{y}}}
$$

It is implied in this equation that the original yarn, once embedded in the fabric matrix made of other yarns, has to be treated as a chain of statistically independent yarn segments of length $l_{\mathrm{c}}$ whose value is determined by the yarn in situ strength, yarn size and the interactions between yarns as specified in the equation.

Furthermore, as indicated by Henstenburg and Phoenix, ${ }^{26}$ the actual fragment lengths are not a constant and vary in the range $l_{\mathrm{c}} / 2$ to $l_{\mathrm{c}}$. This problem can be solved by replacing $l_{\mathrm{c}}$ with the mean fragment length $3 l_{\mathrm{c}} / 4$ if a uniform distribution of this length is assumed. For simplicity however, we still use eqn (10) for the present study.
More importantly, the fragmentation process has revealed that during the extension of the fabric, the yarns are stretched segment by segment through fabric. So, because of the strong strength-length dependence of yarns, the strengths of these yarn segments will become higher owing to their shorter lengths.

Also, during the fabric extension, by definition any yarn fragment with length longer than $l_{\mathrm{c}}$ is still able to break somewhere along its center section as its stress exceeds its current in situ strength, $\sigma_{\mathrm{y}}$. Therefore, the mean length before yarns break into lengths $l_{\mathrm{c}}$ will be $4 l_{\mathrm{c}} / 3$. This will be the length by which the value of $\sigma_{\mathrm{y}}$ for the new yarn fragment is determined. Keeping this in mind and combining eqs (2) and (10) gives:

$$
l_{\mathrm{c}}=\left[\frac{2}{L_{\mathrm{y}} n_{\mathrm{y}} \tau_{\mathrm{y}}}\left(\frac{4}{3} \alpha_{\mathrm{y}}\right)^{-\frac{1}{\beta_{\mathrm{y}}}} \Gamma\left(1+\frac{1}{\beta_{\mathrm{y}}}\right)\right]^{\beta_{\mathrm{y}} /\left(1+\beta_{\mathrm{y}}\right)}
$$

The in situ yarn strength, $\sigma_{y}$, can then be determined from eqn (2) replacing the original yarn length, $l_{\mathrm{y}}$, by the critical yarn length, $l_{c}$, to reflect the yarn-fabric interactions.

To accommodate other weave structures besides the plain weave, the above equation can be rewritten as:

$$
l_{\mathrm{c}}=\left[\frac{1}{C_{\mathrm{y}} n_{\mathrm{y}} \tau_{\mathrm{y}}}\left(\frac{4}{3} \alpha_{\mathrm{y}}\right)^{-\frac{1}{\beta_{\mathrm{y}}}}\left(1+\frac{1}{\beta_{\mathrm{y}}}\right)\right]^{\beta_{\mathrm{y}}\left(1+\beta_{\mathrm{y}}\right)}
$$

where $C_{\mathrm{y}}$ represents the length of the yarn-yarn contact area. In the case of plain weave, we have as above $C_{\mathrm{y}}=L_{\mathrm{y}} / 2$.

\subsection{The components of the shear resistance $\tau_{\mathrm{y}}$ at yarn contact area}

Even with a fabric made of yarns whose weight is so light as to be negligible, a certain force is still required to pull a yarn out of the fabric by overcoming the resistances between the yarn contact points. Yet the magnitude of this pullout force will increase significantly when certain pressure is applied at the contact points. In other words, there exist two components of the shear traction $\tau_{\mathrm{y}}$ which resists any attempt of relative yarn movement at the contact area. Of the two components, one is pressure-independent adhesive force termed $\tau_{\mathrm{y} 2}$, and the other is pressure-related frictional force $\tau_{\mathrm{y} 1}$ governed by the friction law as explained above. That is

$$
\tau_{\mathrm{y}}=\tau_{\mathrm{y} 1}+\tau_{\mathrm{y} 2}
$$

The pressure can either be applied directly to the fabric surface, or be generated from tensile load exerted uniaxially or biaxially to cause a tightening effect to the fabric so as to increase the pressure at the yarn contact points.

Assume the fabric is under a biaxial loading case where the load $\sigma_{\mathrm{L}}$ is applied in the warp direction and the load $\sigma_{\mathrm{T}}$ in the weft direction as seen in Fig. 2(a). 

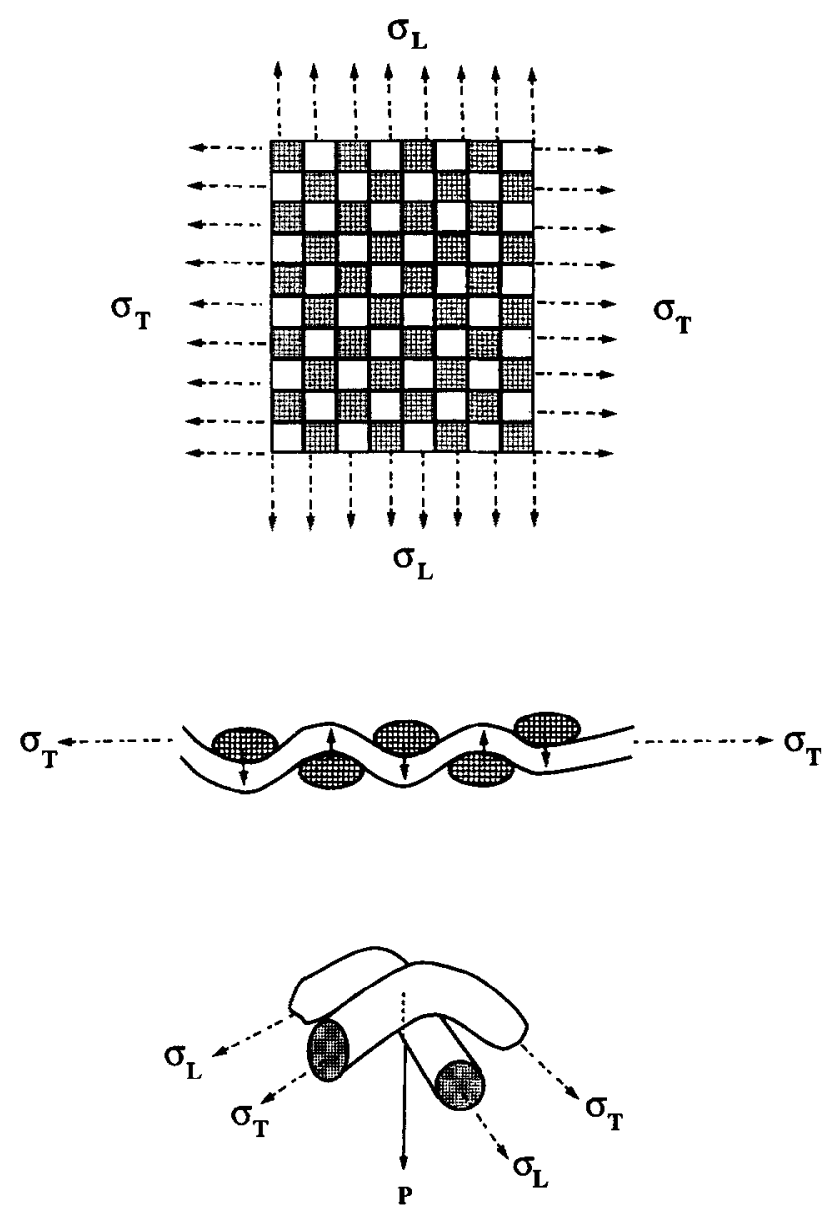

Fig. 2. Various forces on a plain weave fabric. (a) Biaxial loading on the fabric; (b) cross-section along a weft yarn; (c) forces at an interlacing point.

These external loads will create a pressure, $P$, at each contact point shown in Fig. 2(b) and (c) of a cross-section of the fabric.

The compression force, $P$, is exerted on a contact point of two crossing yarns with circumference $L_{\mathrm{y}}$ to cause a frictional force $\mu P$ where $\mu$ is the frictional coefficient. This frictional force is balanced by the shear force $\tau_{\mathrm{y} 1}$ through:

$$
\tau_{\mathrm{y} 1} C_{\mathrm{y}}=\mu P
$$

So the frictional component of the shear resistance is:

$$
\tau_{\mathrm{y} 1}=\frac{\mu P}{C_{\mathrm{y}}}
$$

The resulting normal compression force $P$ as shown in Fig. 2(c) can be calculated as:

$$
P=\left(2 \sigma_{\mathrm{T}} \sin \theta_{\mathrm{T}}+2 \sigma_{\mathrm{L}} \sin \theta_{\mathrm{L}}\right)
$$

where $\theta_{i}(i=\mathrm{T}, \mathrm{L})$ are the interlacing angles at the longitudinal and transverse directions, respectively, and can be expressed from Fig. 1(b) as:

$$
\theta=\arctan \left(\frac{2 t_{\mathrm{y}}}{b_{\mathrm{y}} / 2}\right)=\arctan \left(4 t_{\mathrm{y}} n_{\mathrm{y}}\right)
$$

Note that the between yarn distance $b_{\mathrm{y}}=1 / n_{\mathrm{y}}$.
Since our ultimate goal is to determine the fabric tensile strength in onc, say the warp, direction when the fabric is under a biaxial loading situation, the involvement of the warp stress $\sigma_{\mathrm{L}}$ in eqn (16) brings considerable difficulty for further analysis. To simplify the result, we need to study the ratios $\sigma_{\mathrm{T}} / \sigma_{\mathrm{L}}$ and $\sin \theta_{\mathrm{L}} / \sin \theta_{\tau}$. It is not hard to conceive that when we increase $\sigma_{\mathrm{L}}$, the value of $\theta_{\mathrm{L}}$ will decrease, and a similar connection exists between $\sigma_{\mathrm{T}}$ and $\theta_{\mathrm{T}}$. Supposing an identical geometry in both directions of the fabric, we can therefore assume a relationship:

$$
\frac{\sigma_{\mathrm{T}}}{\sigma_{\mathrm{L}}}=\frac{\sin \theta_{\mathrm{L}}}{\sin \theta_{\mathrm{T}}}
$$

or

$$
\sigma_{\mathrm{T}} \sin \theta_{\mathrm{T}}=\sigma_{\mathrm{L}} \sin \theta_{\mathrm{L}}
$$

So eqn (16) becomes:

$$
P=4 \sigma_{\mathrm{T}} \sin \theta_{\mathrm{T}}
$$

It has to be pointed out, however, in the case of uniaxial extension where $\sigma_{\mathrm{T}}=0$, the value of $P$ will not be zero because of the contribution from the existing force $\sigma_{\mathrm{L}}>0$. Yet, as to be proved later, this contribution will be reduced as the fabric is only tightened in one direction with the load applied, and there is an absence of the lateral extension $\sigma_{\mathrm{T}}$

Combining eqs (15), (17) and (20) yields the first shear resistance component:

$$
\tau_{\mathrm{y} 1}=\frac{4 \mu}{C_{\mathrm{y}}} \sigma_{\mathrm{T}} \sin \left[\arctan \left(4 t_{\mathrm{y}} n_{\mathrm{y}}\right)\right]
$$

The second component of $\tau_{\mathrm{y}}$ can be derived based on the analysis of Pan and Yoon ${ }^{24}$ on the yarn pullout behavior from a fabric. When a yarn is pulled out from a woven fabric, the maximum adhesive surface force $p_{\mathrm{m}}$ at one contact point was derived as:

$$
p_{\mathrm{m}}=\frac{2 \tau_{\mathrm{s}} w_{\mathrm{y}}}{\rho} \tanh 2 \rho w_{\mathrm{y}}
$$

where $\tau_{\mathrm{s}}$ is defined as the elastic shear strength of the contact area, and $\rho$ is a factor reflecting the geometrical and mechanical properties of the yarn:

$$
\rho=\frac{1}{t_{\mathrm{y}}} \sqrt{\frac{G_{\mathrm{y}}}{\pi E_{\mathrm{y}}}}
$$

where $G_{y} / E_{\mathrm{y}}$ is the ratio of the longitudinal shear modulus and tensile modulus of the yarn. Similarly, this force is balanced by the second shear resistance component $\tau_{\mathrm{y} 2}$ as:

$$
\tau_{\mathrm{y} 2} \frac{L_{\mathrm{y}}}{2}=p_{\mathrm{m}}
$$


or

$$
\tau_{\mathrm{y} 2}=\frac{4 \tau_{\mathrm{s}} w_{\mathrm{y}}}{L_{\mathrm{y}} \rho} \tanh 2 \rho w_{\mathrm{y}}=\frac{2 \tau_{\mathrm{s}} w_{\mathrm{y}}}{C_{\mathrm{y}} \rho} \tanh 2 \rho w_{\mathrm{y}}
$$

independent of loading situation and directions.

As shown above, $\tau_{\mathrm{y} 2}$ value is determined by the yarn mechanical properties $G_{\mathrm{y}} / E_{\mathrm{y}}$ and the geometrical property or the ellipticity of the yarn cross-sectional area $w_{\mathrm{y}} / t_{\mathrm{y}}$.

\subsection{The fabric strength as a stochastic variable}

In view of the preceding analysis, the strength in the warp direction of a practical fabric $\sigma_{\mathrm{L}}$ has also to be treated as a probabilistic or stochastic variable and its mean value can be obtained from:

$$
\overline{\sigma_{\mathrm{L}}}=V_{\mathrm{f}} \overline{\sigma_{\mathrm{b}}}\left(l_{\mathrm{c}}\right)
$$

and the standard deviation:

$$
\Theta_{\mathrm{L}}=V_{\mathrm{f}} \Theta_{\mathrm{b}}\left(l_{\mathrm{c}}\right)
$$

where $V_{\mathrm{f}}$ is the overall fiber volume fraction of the fabric, and $\overline{\sigma_{\mathrm{b}}}$ and $\Theta_{\mathrm{b}}$ are the mean yarn bundle strength and its standard deviation which can be calculated from eqns (5) and (6), except that the yarn length $l_{\mathrm{y}}$ in the equations has to be replaced by the critical length $l_{\mathrm{c}}$ defined in eqn (12). This step is crucial so that the effect of yarn-yarn interactions can be included. For convenience, in the following sections, we will use the same format such as $\overline{\sigma_{\mathrm{b}}}\left(l_{\mathrm{c}}\right)$ to designate a strength $\overline{\sigma_{\mathrm{b}}}$ and the length $l_{\mathrm{c}}$ at which the strength is determined.

Then the distribution density function of the fabric strength in the warp direction can be expressed from cqn (4) as:

$$
H\left(\sigma_{\mathrm{L}}\right)=\frac{1}{\sqrt{2 \pi} \theta_{\mathrm{L}}} \exp \left[-\frac{\left(\sigma_{\mathrm{L}}-\overline{\sigma_{\mathrm{L}}}\right)^{2}}{2 \Theta_{\mathrm{L}}^{2}}\right]
$$

\section{THE STRESS/STRAIN CURVE OF A FABRIC}

Suppose the single yarns used for this study are of linear mechanical behavior prior to failure. The yarn properties such as the tensile modulus, yarn surface and cross-sections as well as fabric parameters are provided in Table 1.

For a fabric of length $l_{\mathrm{c}}$ under a given external strain $\epsilon_{\mathrm{L}}$ high enough to cause yarn breakage, the yarns in the fabric will not fail at the same time because of the variations between the yarn strengths. Instead, they will break gradually according to their strength distribution over a certain range of the stress $E_{\mathrm{y}} \epsilon_{\mathrm{L}}$. Therefore, the fabric tensile stress $\sigma_{\mathrm{L}}$ can be expressed as:

$$
\sigma_{\mathrm{L}}=\Psi_{\mathrm{L}}\left(l_{\mathrm{c}}\right) V_{\mathrm{f}} E_{\mathrm{y}} \epsilon_{\mathrm{L}}
$$

Table 1. The assumed property values for calculation

\begin{tabular}{lcc}
\hline Property & $\begin{array}{c}\text { Typical } \\
\text { value }\end{array}$ & Unit \\
\hline Fiber density, $\rho_{\mathrm{f}}$ & $1 \cdot 30$ & $\mathrm{~g} / \mathrm{cm}^{3}$ \\
Fabric fiber volume fraction, $V_{\mathrm{f}}$ & $0 \cdot 5$ & \\
Fabric width, $w_{\mathrm{F}}$ & $25 \cdot 4$ & $\mathrm{~mm}$ \\
Yarn tensile modulus, $E_{\mathrm{y}}$ & $1 \cdot 1$ & $\mathrm{GPa}$ \\
Thread shape parameters, $\beta_{\mathrm{y}}$ & $4 \cdot 0$ & \\
Thread scale parameter, $\alpha_{\mathrm{y}}$ & $5 \cdot 0$ & $\mathrm{GPa}^{-\beta \mathrm{y}} / \mathrm{mm}$ \\
Yarn cross-section ellipticity, $w_{\mathrm{y}} / t_{\mathrm{y}}$ & $1 \cdot 4$ & \\
Inter-yarn frictional coefficient, $\mu$ & $0 \cdot 3$ & \\
Fabric count, $n_{\mathrm{y}}$ & $3 \cdot 9$ & $\mathrm{~mm}^{-1}$ \\
Number of yarns in fabric, $N_{\mathrm{y}}$ & $100 \cdot 0$ & \\
\hline
\end{tabular}

where $E_{\mathrm{y}}$ is the yarn tensile modulus, $V_{\mathrm{f}}$ is the overall fiber volume fraction of the fabric, and $\Psi_{\mathrm{L}}\left(l_{\mathrm{c}}\right)$ is the fraction of the number of warp yarns that are not broken yet at the current stress level $E_{\mathrm{y}} \epsilon_{\mathrm{I}}$ and are still carrying the load. This surviving yarn ratio can be calculated according to Pan: ${ }^{27}$

$$
\Psi_{\mathrm{L}}\left(l_{\mathrm{c}}\right)=\int_{E_{\mathrm{y}} \epsilon_{\mathrm{L}}}^{\infty} H\left(\sigma_{\mathrm{b}}\right) \mathrm{d} \sigma_{\mathrm{b}}
$$

where $H\left(\sigma_{\mathrm{b}}\right)$ is the distribution density function of the yarn bundle strength defined in eqn (4).

\section{CALCUlation AND discussion}

It has to be pointed out that the main focus of the present study is to develop a probabilistic model to predict tensile fabric strength. Therefore, when we apply the existing theories on the fragmentation process in our analysis, we only use the most widely accepted, instead of the most advanced, theoretical results so as to avoid distractions and additional complicities. Our model could, of course, be improved by using it in conjunction with the most recent theories.

\subsection{The yarn cross-sectional dimensions}

In the present case, the parameters describing the yarn cross-section including the yarn width $w_{\mathrm{y}}$ and thickness $t_{\mathrm{y}}$ are the ones that have no convenient and reliable way to be measured. However, they can be replaced with others which are easier to measure or are more familiar in practice.

It is rational to assume:

or

$$
\frac{w_{\mathrm{y}}}{t_{\mathrm{y}}}=a_{1}
$$

$$
w_{\mathrm{y}}=a_{1} t_{\mathrm{y}}
$$

The value of $a_{1}$ has been assumed to range from 1 to 2 based on our experimental observations.

There also exists a relationship:

$$
t_{\mathrm{y}}+w_{\mathrm{y}}=D_{\mathrm{y}}
$$

where $D_{y}$ is the nominal or equivalent diameter of the 
yarns with the assumption of circular yarn crosssections which can be calculated as: ${ }^{4}$

$$
D_{y}(\mathrm{~mm})=2 \sqrt{\frac{\text { tex }}{\pi \rho_{\mathrm{f}} V_{\mathrm{fy}} 10^{5}}} \times 10
$$

where tex is the yarn number, $\rho_{\mathrm{f}}$ is the fiber density in $\mathrm{g} / \mathrm{cm}^{3}$, and $V_{\mathrm{fy}}$ is the fiber volume fraction of the yarn which is different from, usually greater than, the overall fiber volume fraction of the fabric, $V_{f}$, which appeared in eqn (29), because of the existence of the inter-yarn spaces in a fabric. We can solve from above equations the yarn thickness:

$$
t_{\mathrm{y}}(\mathrm{mm})=\frac{2 \sqrt{\frac{\mathrm{tex}}{\pi \rho_{\mathrm{f}} V_{\mathrm{fy}} 10^{5}}}}{1+a_{1}} \times 10
$$

Unless stated otherwise, for fabrics of different fiber types used in this study, by using $\rho_{\mathrm{f}}=1.30 \mathrm{~g} / \mathrm{cm}^{3}$ for acetate fiber, $1.38 \mathrm{~g} / \mathrm{cm}^{3}$ for PET and $1.51 \mathrm{~g} / \mathrm{cm}^{3}$ for cotton fibers, ${ }^{28}$ and assuming identical values $a_{1}=1.4$, and $V_{\mathrm{fy}}=0.628$, we thus calculated their $t_{\mathrm{y}}$ values.

\subsection{Determination of the yarn-yarn adhesion}

The shear resistance at a yarn-yarn crossing point has been proved to be:

$$
\begin{aligned}
\tau_{\mathrm{y}}= & \tau_{\mathrm{y} 1}+\tau_{\mathrm{y} 2}=\frac{4 \mu}{C_{\mathrm{y}}} \sigma_{\mathrm{T}} \sin \left[\arctan \left(4 t_{\mathrm{y}} n_{\mathrm{y}}\right)\right] \\
& +\frac{2 \tau_{\mathrm{s}} w_{\mathrm{y}}}{C_{\mathrm{y}} \rho} \tanh 2 \rho w_{\mathrm{y}}
\end{aligned}
$$

The frictional component $\tau_{\mathrm{y} 1}$ is dependent on the external pressure, whereas the adhesive component $\tau_{\mathrm{y} 2}$ is entirely determined by the surface geometry and properties of the yarns, and is hence an intrinsic system property irrelevant to the external loadings. Furthermore, the force $\tau_{\mathrm{y} 2}$, or the elastic shear strength of the contact area $\tau_{\mathrm{s}}$, can be determined theoretically.

Assume there is no external load or pressure exerted at the yarn-yarn contact arca so that $\tau_{\mathrm{y} 1}=0$ and we have the shear traction as:

$$
\tau_{\mathrm{y}}=\tau_{\mathrm{y} 2}=\frac{2 \tau_{\mathrm{s}} w_{\mathrm{y}}}{C_{\mathrm{s}} \rho} \tanh 2 \rho w_{\mathrm{y}}
$$

If we then pull a yarn out of the fabric, the critical yarn length at which the yarn will break within the fabric rather than be pulled out is still $l_{c}$ defined in eqn (12), but at the condition $\tau_{y}=\tau_{y 2}$. The force equilibrium on the yarn at breaking becomes:

$$
\tau_{\mathrm{y} 2} C_{\mathrm{y}} n_{\mathrm{y}} l_{\mathrm{c}}=\widetilde{\sigma_{\mathrm{y}}}\left(l_{\mathrm{c}}\right)=\left(l_{\mathrm{c}} \alpha_{\mathrm{y}} \beta_{\mathrm{y}}\right)^{-\frac{1}{\beta_{\mathrm{y}}}} \exp \left(-\frac{1}{\beta_{\mathrm{y}}}\right)
$$

Since all other variables can be considered as given, the value of $\tau_{\mathrm{y} 2}$ or $\tau_{\mathrm{s}}$ can be obtained by solving the above equation. It is found during the calculation, however, that the magnitudes of both $\tau_{\mathrm{y} 2}$ and $\tau_{\mathrm{s}}$ are extremely small. Therefore, their effects on the following calculations are in fact negligible. In other words, between the two shear resistance components, the frictional component is far more dominant than the adhesive one when external load exists. To simplify the process, we use in the following calculations:

$$
\tau_{\mathrm{y}} \approx \tau_{\mathrm{y} 1}
$$

This shear force $\tau_{y}$ is in fact a reflection of the 'shear bonding strength' of the yarn-yarn contact point, and is related to yarn surface frictional behavior, and the transverse tensile load $\sigma_{\mathrm{T}}$ as indicated in eqn (21). Figure 3 depicts the relationship between $\tau_{\mathrm{y} 1}$ and the variables involved. For easy comparison with experimental results later, the transverse tensile stress $\sigma_{\mathrm{T}}$ is expressed as $\mathrm{N}$ per thread, and the unit for $\tau_{\mathrm{y} 1}$ follows as $\mathrm{N}$ per contact point. Here the yarn number tex $=40.0$ and inter-yarn frictional coefficient $\mu=0.3$ are used in the figure.

As indicated in eqn (21), the value of $\tau_{y 1}$ increases linearly with the transverse tensile load $\sigma_{\mathrm{T}}$. A flatter yarn cross-section, represented by a higher ellipticity of the yarn cross-sectional area, $w_{\mathrm{y}} / t_{\mathrm{y}}$, will result in a lower shear force $\tau_{\mathrm{y} 1}$, when the thread count $n_{\mathrm{y}}$ and the fiber volume fraction of the yarn $V_{\mathrm{fy}}$ are given as shown in Fig. 3(a). The effects of both $V_{\mathrm{fy}}$ and $n_{\mathrm{y}}$, on the other hand, can be seen in Fig. 3(b) where $\sigma_{\mathrm{T}}$ and $w_{\mathrm{y}} / t_{\mathrm{y}}$ are given. It is indicated in this figure that a higher $V_{\mathrm{fy}}$ or $n_{\mathrm{y}}$, i.e. more fibers in a yarn or more yarns per unit fabric length, will lead to a higher $\tau_{\mathrm{y} 1}$ value. However, $n_{\mathrm{y}}$ has a more substantial effect on $\tau_{\mathrm{y} 1}$ when $V_{\mathrm{fy}}$ is greater.

In summary, at a given level of the transverse load $\sigma_{\mathrm{T}}$, for a less flat yarn, a denser fabric will result in a higher shear traction $\tau_{y 1}$, or a stronger 'bond' at the contact point between yarns.

\subsection{The critical yarn length $\boldsymbol{l}_{\mathrm{c}}$}

Because of the mutual restraints betwecn the warp and weft yarns, these yarns in a tensioned fabric will each behave as a series of sub-bundle systems made of links of length $l_{\mathrm{c}}$ whose value is determined from eqn (11) by the mechanical and geometrical properties of the yarn and the fabric system. A relative scale $l_{\mathrm{c}} / l_{\mathrm{y}}$ is used here, where $l_{\mathrm{y}}=152.4 \mathrm{~mm}$ is the original yarn (fabric) length. Note that the effect of the length discrepancy between a fabric and a yarn from the fabric, which is caused by yarn crimping within the fabric, is neglected here, since it has been dealt with in detail elsewhere. ${ }^{16}$

The ratio $l_{c} / l_{\mathrm{y}}$ is an indicator of the interactions between yarns during fabric extension, and a smaller $l_{\mathrm{c}} / l_{\mathrm{y}}$ value reveals a greater reinforcing effect due to 

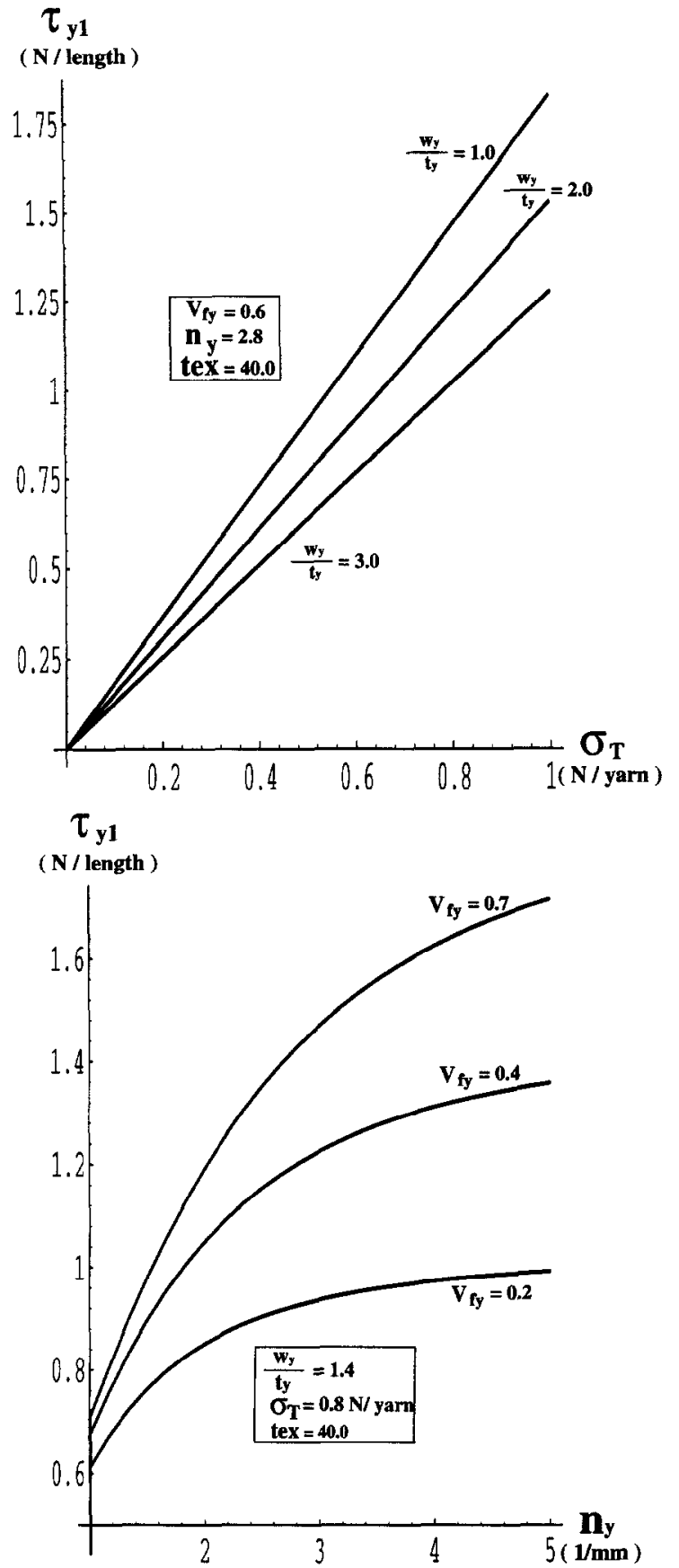

Fig. 3. Frictional component, $\tau_{\mathrm{y} 1}$ of the shear force at a interlacing point: (a) $\tau_{\mathrm{y} 1}$ versus transverse tension, $\sigma_{\mathrm{T}}$, at three levels of ellipticity $w_{\mathrm{y}} / t_{\mathrm{y}}$ yarn cross-section; (b) $\tau_{\mathrm{y} 1}$ versus fabric count, $n_{\mathrm{y}}$, at three levels of fiber volume fraction, $V_{\mathrm{fy}}$, of yarn.

the yarn-yarn interactions in a tensioned fabric. The key factor for the determination of $l_{\mathrm{c}} / l_{\mathrm{y}}$ is the "shear bonding strength' $\tau_{\mathrm{y}}$ at the yarn interlacing points.

Figure 4 illustrates the relationship between $l_{\mathrm{c}} / l_{\mathrm{y}}$ and the transverse load $\sigma_{\mathbf{T}}$ at different levels of the yarn Weibull parameters. In general, the ratio decreases at increasing transverse load $\sigma_{\mathrm{T}}$. This relationship is also influenced by the values of the Weibull parameters of the yarn. For example, a
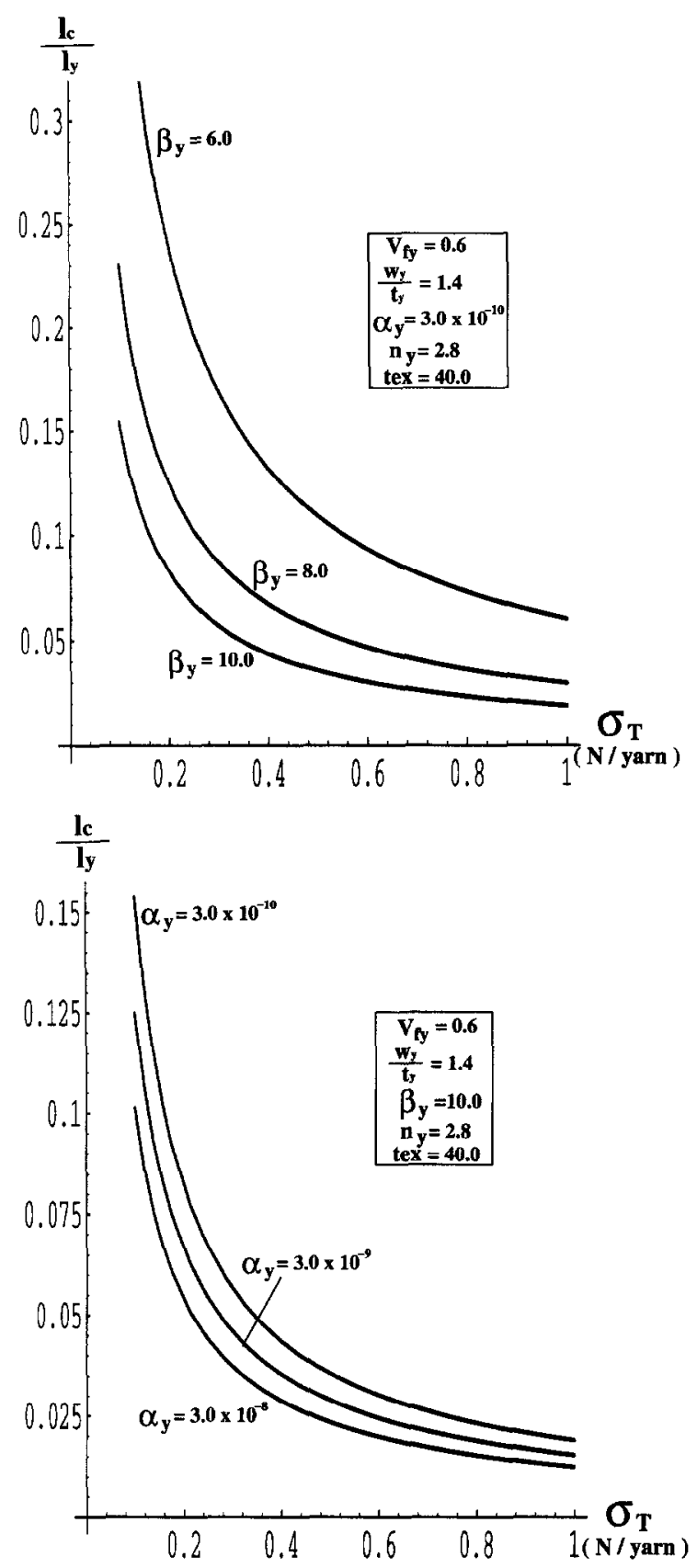

Fig. 4. Effects of variables on $l_{\mathrm{c}} / l_{\mathrm{y}}$ ratio: (a) $l_{\mathrm{c}} / l_{\mathrm{y}}$ versus transverse tension, $\sigma_{\mathrm{T}}$, at three levels of shape parameter, $\beta_{\mathrm{y}}$, of yarn strength; (b) $l_{\mathrm{c}} / l_{\mathrm{y}}$ versus transverse tension, $\sigma_{\mathrm{T}}$, at three levels of scale parameter, $\alpha_{\mathrm{y}}$, of yarn strength.

smaller value of the shape parameter $\beta_{y}$ of the yarn strength, meaning a thread with greater strength variation, will lessen the reinforcing effect, leading to a higher $l_{c} / l_{\mathrm{y}}$ value as seen in Fig. 4(a), whereas in Fig. 4(b) a greater scale parameter $\alpha_{\mathrm{y}}$ will intensify the interactions and enhancement resulting in a smaller $l_{\mathrm{c}} / l_{\mathrm{y}}$ value.

On the other hand, it is clear that the mean fabric strength per yarn would be greater than the mean yarn strength only when the ratio $l_{c} / l_{y}<1$; this is in fact the critical condition for the fragmentation 
process to occur. It in turn defines the critical values for all other variables involved in order for the reinforcing mechanism due to yarn-yarn interactions at the interlacing points to function. For instance, this critical condition

$$
\frac{l_{\mathrm{c}}}{l_{\mathrm{y}}}=1
$$

can be used to define the minimum value of the transverse tensile load $\sigma_{\mathrm{T}}$ in order to yield a stronger
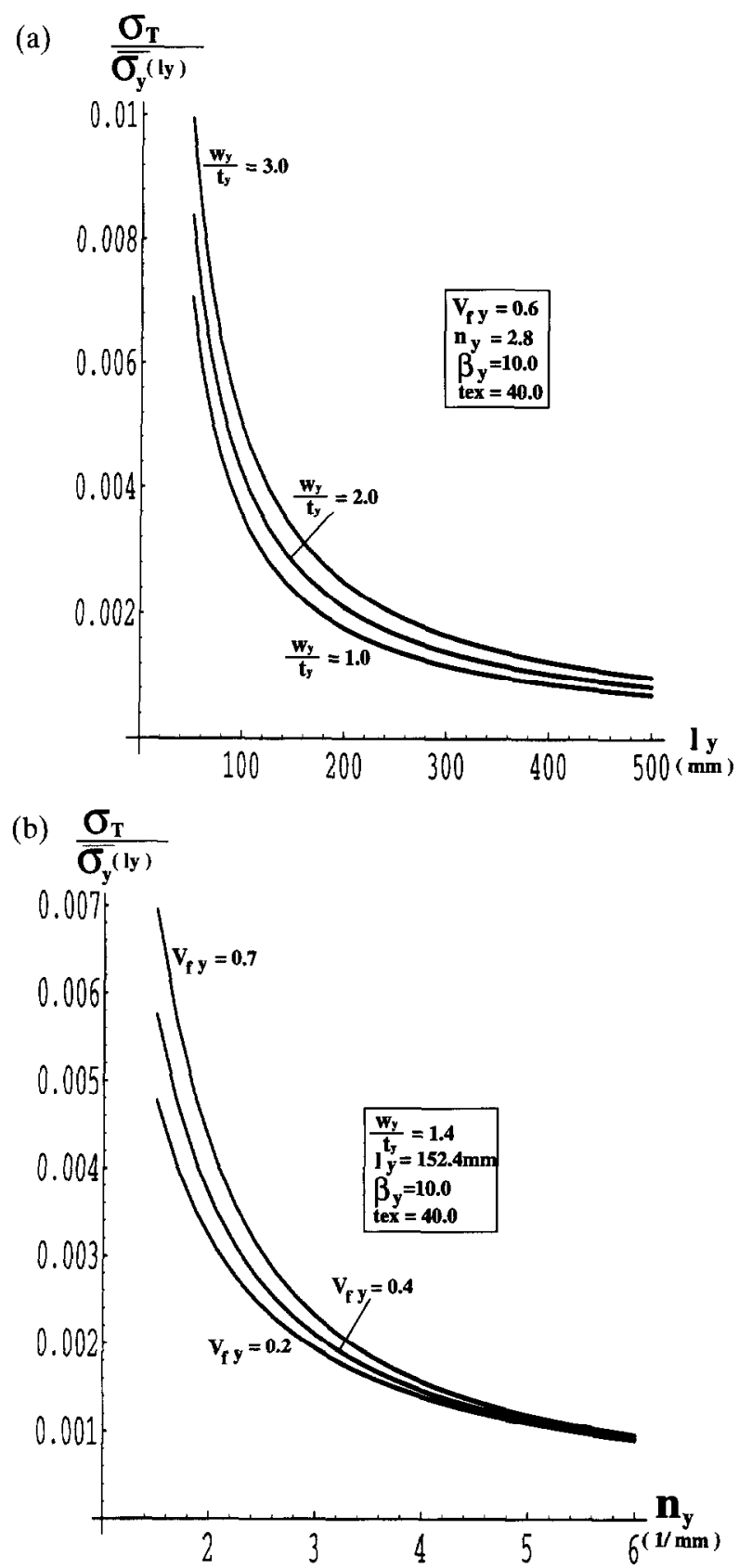

Fig. 5. The critical value $\sigma_{\mathrm{T}} / \overline{\sigma_{\mathrm{y}}}\left(l_{\mathrm{y}}\right)$ ratio: (a) $\sigma_{\mathrm{T}} / \overline{\sigma_{\mathrm{y}}}\left(l_{\mathrm{y}}\right)$ versus original yarn length, $l_{\mathrm{y}}$, at three levels of ellipticity $w_{y} / t_{y}$ of yarn cross-section; (b) $\sigma_{\mathrm{T}} / \overline{\sigma_{\mathrm{y}}}\left(l_{\mathrm{y}}\right)$ versus fabric count, $n_{\mathrm{y}}$, at three levels of fiber volume fraction, $V_{\mathrm{fy}}$, of yarn; (c) $\sigma_{\mathrm{T}} / \sigma_{\mathrm{y}}\left(l_{\mathrm{y}}\right)$ versus shape parameter, $\beta_{\mathrm{y}}$, of yarn at three levels of inter-yarn frictional coefficient $\mu$.

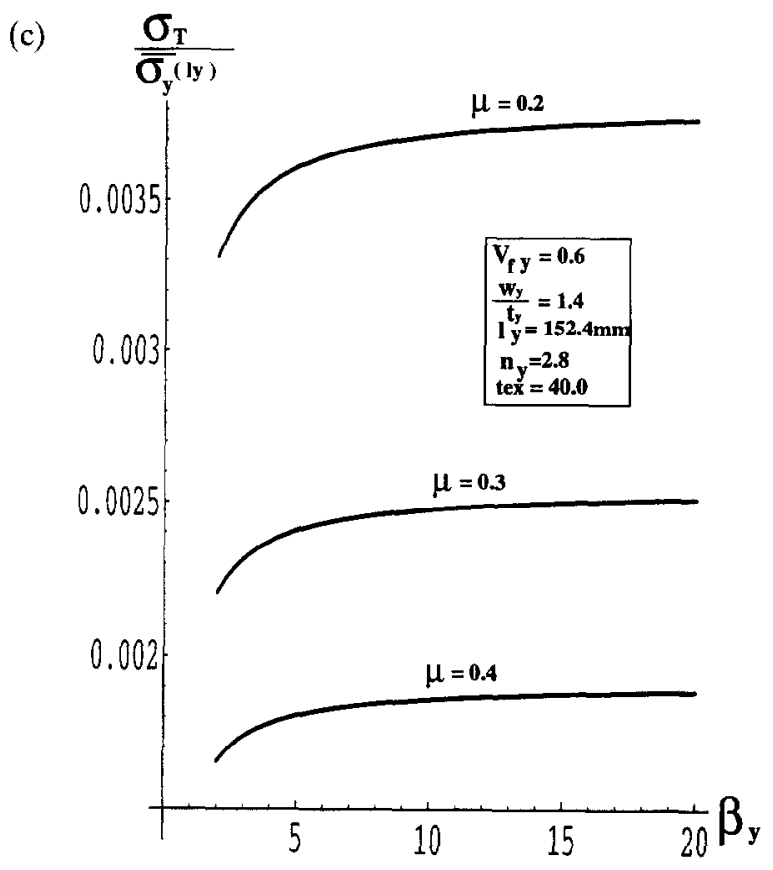

Fig. 5. (Continued.)

fabric. From this condition we can find the solution in relative scale $\sigma_{\mathrm{T}} /\left(\sigma_{\mathrm{y}}\left(l_{\mathrm{y}}\right)\right)$ by combining eqns (2), (12) and (21) with some mathematical manipulations:

$$
\frac{\sigma_{\mathrm{T}}}{\overline{\sigma_{\mathrm{y}}}\left(l_{\mathrm{y}}\right)}=\left(\frac{3}{4}\right)^{\frac{1}{\beta_{\mathrm{y}}}} \frac{1}{4 \mu \sin \left[\arctan \left(4 t_{\mathrm{y}} n_{\mathrm{y}}\right)\right] l_{\mathrm{y}} n_{\mathrm{y}}}
$$

where $\overline{\sigma_{y}}\left(l_{y}\right)$ is the mean yarn strength at original length $l_{\mathrm{y}}$ from eqn (2).

Figure 5 is thus constructed using the above equation to provide the critical levels of all related variables under which the mean fabric strength per yarn will be greater than the mean yarn strength. It is seen from the figure that, when all other conditions are given, a longer original yarn length $l_{\mathrm{y}}$, or a less flat yarn cross-section with smaller $w_{y} / t_{y}$ from Fig. 5(a), or a higher fabric count $n_{\mathrm{y}}$ or a smaller yarn fiber volume fraction $V_{\mathrm{fy}}$ in Fig. 5(b), as well as a higher value of the inter-yarn frictional coefficient $\mu$ in Fig. 5(c) will require a lower transverse tensile load $\sigma_{\mathrm{T}}$ to trigger the fragmentation process. It is secn in Fig. 5(b) that at very high fabric count, $n_{\mathrm{y}}$, the yarn fiber volume fraction will have little influence. It is also shown in Fig. 5(c) that the shape parameters of the yarn strength, $\beta_{y}$, once exceeding a certain value, around 5 in the figure, will have a much less significant effect on the required value of $\sigma_{\mathrm{T}} / \overline{\sigma_{\mathrm{y}}}\left(l_{\mathrm{y}}\right)$.

\subsection{The reinforcing effect of yarn-yarn interactions on fabric strength}

As stated above, the mean strength of a bundle of yarns will be lower than the mean strength of its constituent yarns due chiefly to the statistical variations of the yarn strengths between the individual 
yarns. On the other hand, once the bundles are interlaced with each other to form a fabric, upon tensioning of the fabric, the interactions at the interlacing points between yarns will considerably alter the yarn behavior from its ex situ state, and as a result, the so-called fragmentation process will occur which will increase the mean strength of the fabric. The ultimate value of the fabric strength thus becomes a result of these two competing factors. Since eqns (2) and (5) already provide the results showing the influences of the first factor, we need to design a method where only the second factor, namely the effects of yarn-yarn interactions in a tensioned fabric, remains to be effective so as to show its effects. One convenient way to do this is to examine the ratio $\overline{\sigma_{\mathrm{L}}}\left(l_{\mathrm{c}}\right) / \overline{\sigma_{\mathrm{L}}}\left(l_{\mathrm{y}}\right)$ where $\overline{\sigma_{\mathrm{L}}}\left(l_{\mathrm{c}}\right)$ and $\overline{\sigma_{\mathrm{L}}}\left(l_{\mathrm{y}}\right)$ are the fabric strengths with and without inclusion of the fragmentation effects; both of them are calculated from eqn (26), except that the critical yarn length $l_{c}$ and the original yarn length $l_{\mathrm{y}}$ are used, respectively. Since eqn (26) is derived from the result of the mean strength for yarn bundles, the effect of the strength variations between individual yarns is thus excluded in the ratio $\bar{\sigma}_{\mathrm{L}}\left(l_{c}\right) / \bar{\sigma}_{\mathrm{L}}\left(l_{\mathrm{y}}\right)$.

This fabric strength ratio is then plotted against the lateral extension $\sigma_{\mathrm{T}}$ in Fig. 6 . It can be readily proved that:

$$
\frac{\overline{\sigma_{\mathrm{L}}}\left(l_{\mathrm{c}}\right)}{\overline{\overline{\sigma_{\mathrm{L}}}\left(l_{\mathrm{y}}\right)}}=\left(\frac{l_{\mathrm{y}}}{l_{\mathrm{c}}}\right)^{\frac{1}{\beta_{y}}}
$$

The effects of the Weibull scale parameter of the yarn strength $\alpha_{\mathrm{y}}$ on the ratio $l_{\mathrm{c}} / l_{\mathrm{y}}$ is already illustrated in Fig. 4(b) and is therefore excluded in the following figures. We have to retain, however, the shape parameter $\beta_{\mathrm{y}}$ in our discussion because of its involvement in the expression $\left(l_{\mathrm{y}} / l_{\mathrm{c}}\right)^{\frac{1}{\beta_{y}}}$.

First of all, it is seen from all the curves in Fig. 6 that the value $\overline{\sigma_{\mathrm{L}}}\left(l_{\mathrm{c}}\right) / \overline{\sigma_{\mathrm{L}}}\left(l_{\mathrm{y}}\right)$ is always greater than one, and increases as the lateral tension $\sigma_{\mathrm{T}}$ gets greater, indicating that the yarn-yarn interactions always enforce the fabric structure, and the reinforcing mechanism becomes more significant as the interactions intensify; the tensile strength of the fabric thus grows higher and higher with an increasing $\sigma_{\mathrm{I}}$ value.

On the other hand, the impact of $\sigma_{\mathrm{T}}$ on $\overline{\sigma_{\mathrm{L}}}\left(l_{\mathrm{c}}\right) / \overline{\sigma_{\mathrm{L}}}\left(l_{\mathrm{y}}\right)$ is also dependent on the levels of other variables. The effects of the shape parameter $\beta_{\mathrm{y}}$ of yarn strength are shown in Fig. 6(a): a higher $\beta_{\mathrm{y}}$ value results in a lower $\overline{\sigma_{\mathrm{L}}}\left(l_{\mathrm{c}}\right) / \overline{\sigma_{\mathrm{L}}}\left(l_{\mathrm{y}}\right)$ value. In addition, at a given $\sigma_{\mathrm{T}}$ level, a fabric with more yarns per unit length, i.e. a higher $n_{y}$ value in Fig. $6(\mathrm{~b})$, or yarns with less flat cross section of a smaller $w_{\mathrm{y}} / t_{\mathrm{y}}$ value in Fig. $6(c)$, or a higher inter-yarn frictional coefficient $\mu$ in Fig. 6(d), or a less dense yarn with a lower $V_{\mathrm{fy}}$ value in Fig. 6(e) as well as a thicker yarn with a higher yarn number tex in Fig. 6(f) will all result a stronger fabric compared to the mean fabric strength $\overline{\sigma_{\mathrm{L}}}\left(l_{\mathrm{y}}\right)$ ignoring the yarn-yarn interactions.

\subsection{The stress/strain curves of fabric under uniaxial and biaxial loadings}

The stress/strain curves of a plain weave fabric under uniaxial and biaxial loadings can be obtained using eqns (29) and (30) and the data in Table 1 by setting the transverse tension $\sigma_{\mathrm{T}}$ at zero and at different levels. Figure 7 shows the results. Note the vertical axis in the figure is expressed in a relative scale of $\sigma_{\mathrm{L}} / E_{\mathrm{y}}$, where $E_{\mathrm{y}}$ is the yarn modulus whose value is provided in Table 1. It can be concluded from Fig. 7 that increasing the transverse tension $\sigma_{\mathrm{T}}$ will yield both a higher breaking strain and a higher strength for the fabric. It will not, however, affect the modulus of the fabric. Apparently, some of the mechanisms at the initial stage of fabric tensioning such as decrimping ${ }^{4}$ are excluded from the model so that the predictions deviate from the real situations at the initial part of the stress/strain curves.

\subsection{The effects of weave structures}

All the preceding equations are derived based on a plain weave structure. The different weaves will affect the nature of the crossing points, i.c. the actual yarn-yarn contact area and the pressure between the yarns per contact. However, from eqns (12), (21) and (39), we found that the yarn-yarn contact length (area) $C_{\mathrm{y}}$ is cancelled when calculating the yarn critical length $l_{c}$, and hence has no net effect on fabric strength.

An idealized illustration of major weave structures is provided in Fig. 8. Through some simple geometrical and mechanistic analyses and based on the plain weave case dealt with previously, for a $2 / 1$ twill in Fig. 8(a), we can obtain the approximate compression force per yarn-yarn contact point:

$$
P=\frac{8}{3} \sigma_{\mathrm{T}} \sin \theta_{\mathrm{T}}
$$

For a 3/1 twill weave in Fig. 8(b), we have:

$$
P=\frac{10}{4} \sigma_{\mathrm{T}} \sin \theta_{\mathrm{T}}=2 \cdot 5 \sigma_{\mathrm{T}} \sin \theta_{\mathrm{T}}
$$

For a 4/1 satin illustrated in Fig. 8(c), we can prove:

$$
P=\frac{12}{5} \sigma_{\mathrm{T}} \sin \theta_{\mathrm{T}}=2 \cdot 4 \sigma_{\mathrm{T}} \sin \theta_{\mathrm{T}}
$$

Bringing a specific $P$ expression into eqns (20) and then (21) will yield the results corresponding to the weave structure. 
(a) $\frac{\overline{\sigma_{L}\left(l_{c}\right)}}{\overline{\sigma_{L}\left(l_{y}\right)}}$

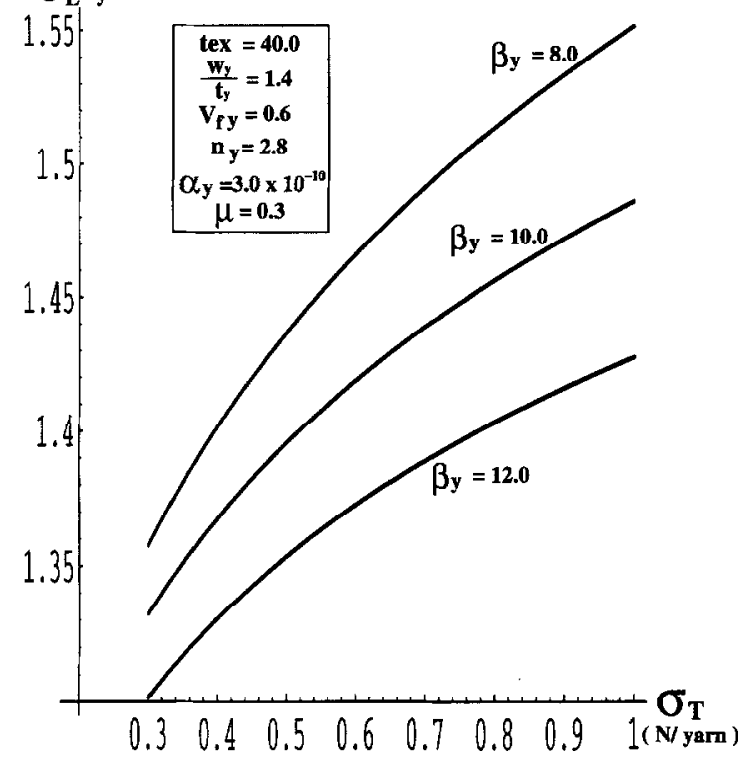

(c) $\frac{\overline{\sigma_{\mathfrak{L}}\left(1_{c}\right)}}{\overline{\sigma_{V^{(1)}}}}$

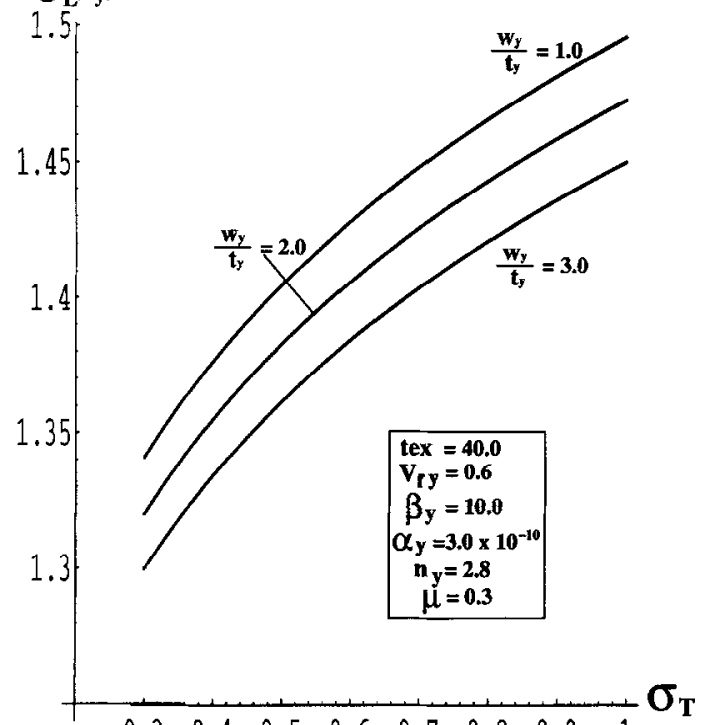

(b) $\frac{\overline{\sigma_{\mathbf{L}}\left(\mathbf{l}_{\mathbf{c}}\right)}}{\overline{\bar{\sigma}_{\mathrm{L}}\left(\mathbf{l}_{\mathbf{y}}\right)}}$

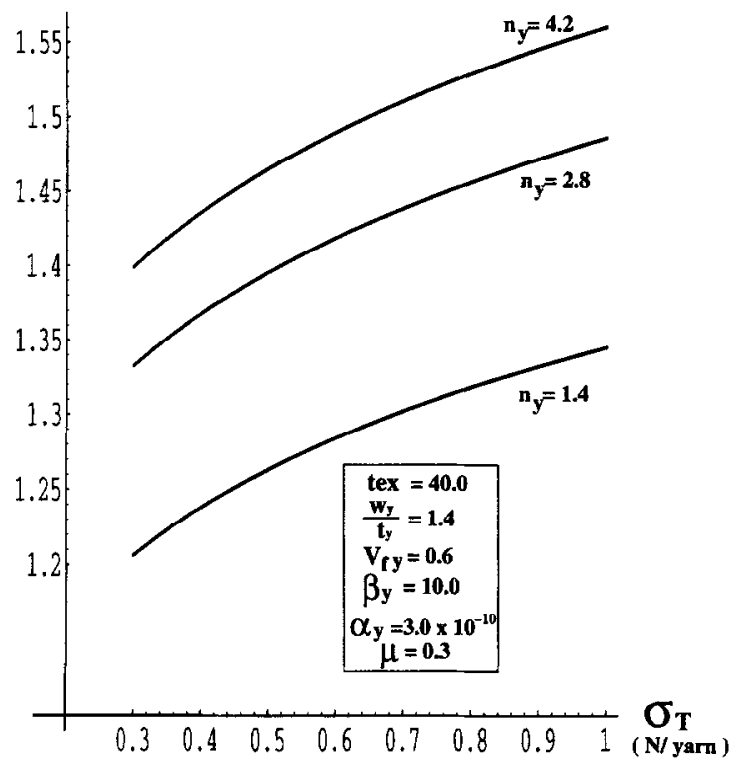

(d) $\frac{\overline{\sigma_{L}\left(l_{c}\right)}}{\overline{\sigma_{L}\left(1_{y}\right)}}$

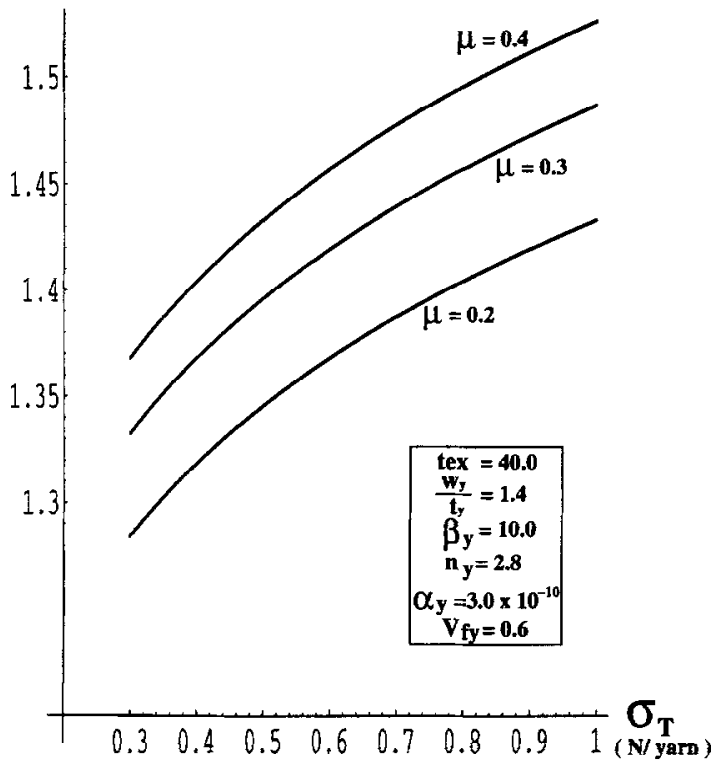

Fig. 6. The strength ratio $\overline{\sigma_{\mathrm{L}}}\left(l_{c}\right) / \overline{\sigma_{\mathrm{L}}}\left(l_{\mathrm{y}}\right)$ versus transverse tension, $\sigma_{\mathrm{T}}$, at three levels of: (a) shape parameter, $\beta_{\mathrm{y}}$, of yarn strength; (b) fabric count $n_{y}$; (c) ellipticity, $w_{y} / t_{y}$, of yarn cross-section; (d) inter-yarn frictional coefficient $\mu$; (e) fiber volume fraction, $V_{\mathrm{fy}}$, of yarn; (f) yarn number (tex).

\subsection{Comparisons between the predicted and tested fabric tensile strengths}

First, we compare the predictions of the fabric mean strength using eqn (26), to the results reported by Shahpurwala and Schwartz. ${ }^{16}$ The fabric samples used by them ${ }^{16}$ are introduced in Table 2 with their $t_{\mathrm{y}}$ values calculated from eqn (35), and the corresponding Weibull parameters of the yarn strength are reproduced in Table 3. Note that the scale parameter, designated as $\alpha_{y 1}$ in Table 3 was defined slightly differently in Ref. 16 from the one, $\alpha_{\mathrm{y} 2}$, used in eqn
(1) in this analysis. Yet the conversion between the two can be easily accomplished using the relationship:

$$
\alpha_{\mathrm{y} 2}=\frac{\left(\alpha_{\mathrm{y} 1}\right)^{-\beta_{y}}}{l_{\mathrm{y}}}
$$

According to Ref. 16, the yarn length $l_{\mathrm{y}}=152 \cdot 4 \mathrm{~mm}$. So the calculated $\alpha_{\mathrm{y} 2}$ values are also listed in Table 3 . It is worth noticing that the physical meaning of $\alpha_{\mathrm{y} 1}$ is the stress lcvel at which $63 \cdot 2 \%$ of the yarns will break. Since there is usually $\alpha_{\mathrm{y} 1}>\overline{\sigma_{\mathrm{y}}}\left(l_{\mathrm{y}}\right)$, meaning no more 
(e) $\frac{\overline{\sigma_{\mathrm{L}}\left(\mathbf{l}_{\mathrm{c}}\right)}}{\overline{\bar{\sigma}_{\mathrm{L}}\left(\mathbf{l}_{\mathrm{y}}\right)}}$

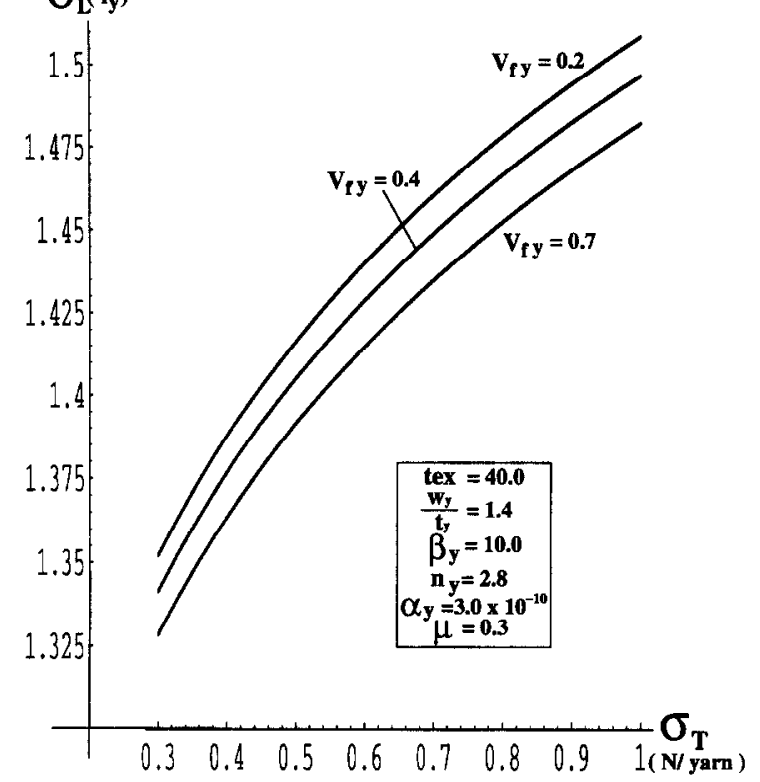

(f)
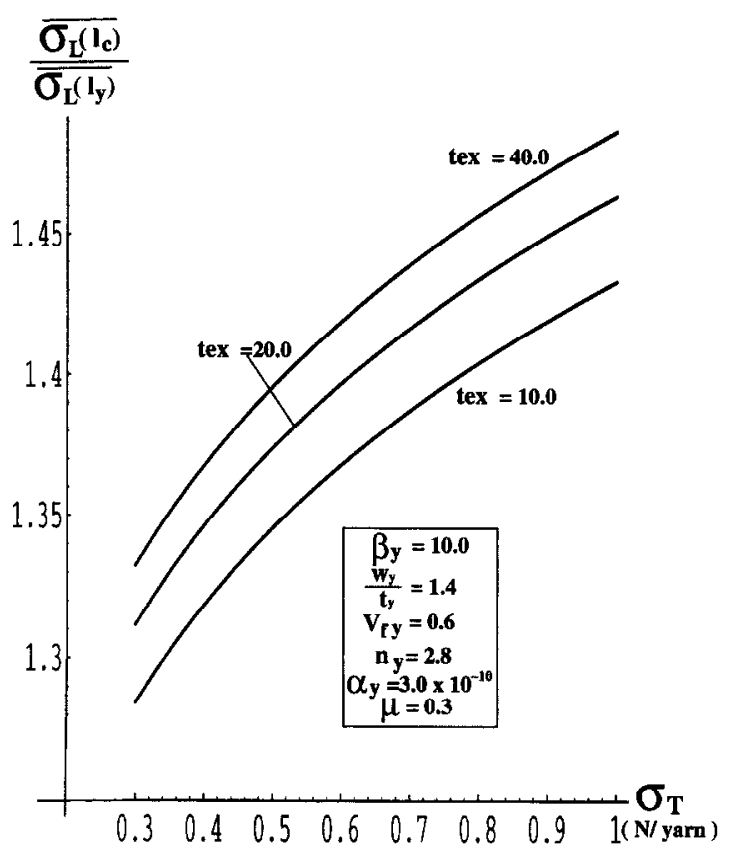

Fig. 6. (Continued.)

than $63.2 \%$ of the yarns will fail at the stress level equal to the mean yarn strength $\overline{\sigma_{y}}\left(l_{\mathrm{y}}\right)$ of eqn (2).

Table 4 provides comparisons between the predicted results by Shahpurwala and Schwartz, ${ }^{16}$ and by the present author, as well as the actually measured fabric strengths. The strength unit in Table 4 is $N$ per yarn. In the table, the results by the present model represent the predictions using eqn (26). Note that

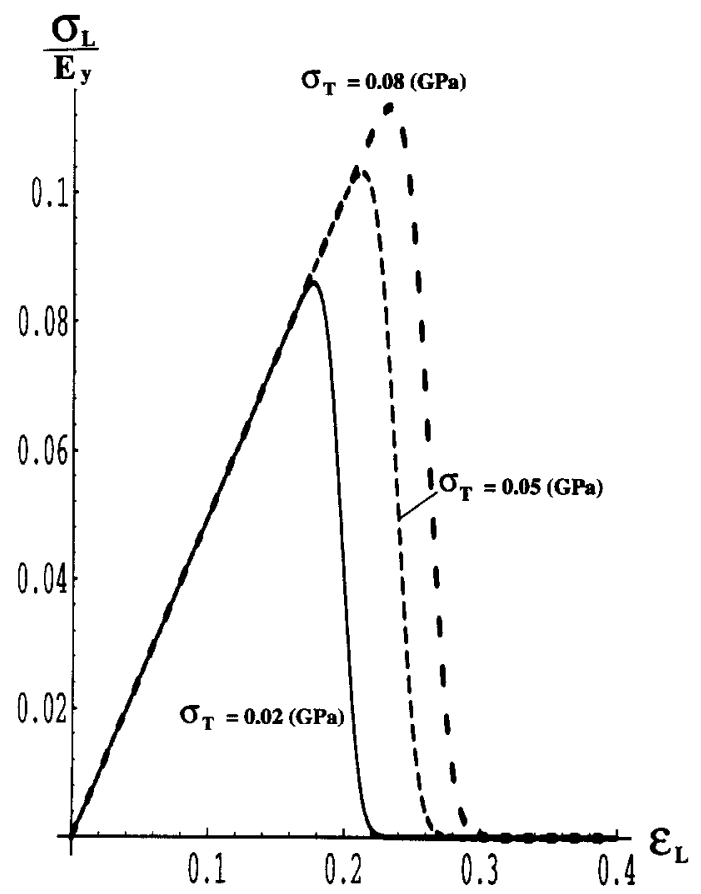

Fig. 7. The stress/strain curves of a plain weave fabric at various levels of transverse tension $\sigma_{\mathrm{T}}$.

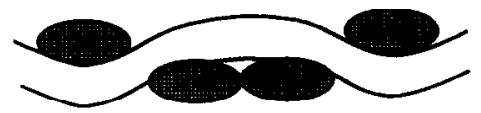

( a )

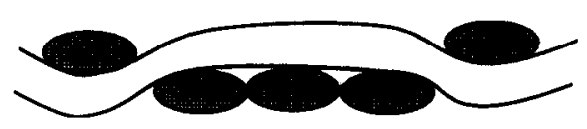

(b)

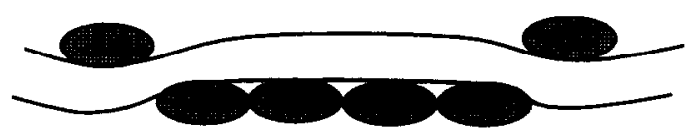

(c)

Fig. 8. The geometry of interlacing points for various weaves: (a) $2 / 1$ twill; (b) $3 / 1$ twill; (c) $4 / 1$ satin. 
Table 2. Fabrics and their parameters from Ref. 16 and $t_{y}$ values

\begin{tabular}{|c|c|c|c|}
\hline Property & $\begin{array}{l}\text { Fabric count, } n_{\mathrm{y}} \\
\quad(\text { thread } / \mathrm{mm})\end{array}$ & $\begin{array}{l}\text { Yarn size } \\
\quad(\text { tex })\end{array}$ & $\begin{array}{c}t_{y} \\
\left(10^{-2}\right. \\
\mathrm{mm})\end{array}$ \\
\hline \multicolumn{4}{|l|}{ 1. Cotton plain } \\
\hline Warp & $4 \cdot 5$ & 27 & $7 \cdot 92$ \\
\hline Weft & $1 \cdot 7$ & 42 & $9 \cdot 88$ \\
\hline \multicolumn{4}{|l|}{$\begin{array}{l}\text { 2. Cotton twill } \\
3 / 1\end{array}$} \\
\hline Warp & $4 \cdot 4$ & 39 & $9 \cdot 52$ \\
\hline Weft & $2 \cdot 1$ & 35 & $9 \cdot 01$ \\
\hline \multicolumn{4}{|l|}{ 3. Cotton satin } \\
\hline Warp & $3 \cdot 9$ & 32 & $8 \cdot 62$ \\
\hline Weft & $2 \cdot 0$ & 39 & $9 \cdot 52$ \\
\hline \multicolumn{4}{|c|}{ 4. Polyester plain } \\
\hline Warp & $3 \cdot 9$ & 5 & $3 \cdot 57$ \\
\hline Weft & $2 \cdot 5$ & 20 & $7 \cdot 13$ \\
\hline
\end{tabular}

since the results are expressed as $N$ per yarn, the fiber volume fraction of the fabric no longer plays a role in the calculation and should be set as unity. The uncorrected results in Table 4 were calculated based on the model of Daniels and the corrected results were based on a modified model of Smith and McCartney. ${ }^{29}$

The data in Table 4 from Ref. 16 are the fabric strengths under uniaxial loading situation, i.e. when $\sigma_{\mathrm{T}}=0$. As we already learned that because of the contribution to the yarn-yarn normal compression force $P$ from the longitudinal external force $\sigma_{\mathrm{L}}$ as shown in eqn (16), the frictional shear resistance $\tau_{\mathrm{y} 1}>0$ even when the transverse tension $\sigma_{\mathrm{T}}=0$. However, in the case of uniaxial loading, the contribution of $\sigma_{\mathrm{L}}$ towards $\tau_{\mathrm{y} 1}$ will be reduced because when the lateral tension $\sigma_{\mathrm{T}}$ is not present, the fabric is not as tight as a biaxial tensioning case, so that the effective normal compression force due to $\sigma_{\mathrm{L}}$ will be discounted by a factor $\Lambda$. It is difficult to

Table 3. Yarn Weibull parameters for fabrics in Table 2

\begin{tabular}{lrrl}
\hline \multicolumn{1}{c}{ Parameter } & \multicolumn{1}{c}{$\beta_{\mathrm{y}}$} & $\alpha_{\mathrm{y} 1}, N$ & $\alpha_{\mathrm{y} 2}, N \beta_{\mathrm{y} / \mathrm{mm}}$ \\
\hline 1. Cotton plain & & & \\
Warp & 10.08 & 5.35 & $2.99 \times 10^{-10}$ \\
Weft & 10.33 & 8.38 & $1.91 \times 10^{-12}$ \\
2. Cotton twill 3/1 & & & \\
Warp & 6.15 & 7.43 & $2.89 \times 10^{-8}$ \\
Weft & 8.75 & 7.19 & $2.09 \times 10^{-10}$ \\
3. Cotton satin 4/1 & & & \\
Warp & 10.25 & 5.31 & $2.43 \times 10^{-10}$ \\
Weft & 9.88 & 6.57 & $5.49 \times 10^{-11}$ \\
4. Polyester plain & 19.02 & 1.77 & $1.26 \times 10^{-7}$ \\
Warp & 10.64 & 5.65 & $6.54 \times 10^{11}$ \\
Weft &
\end{tabular}

determine the value of $\Lambda$ theoretically. An estimated value based on an empirical method can be obtained. For brevity, $\Lambda=0.3$ is selected for this study. Also, for non-plain weave fabric number 2 and 3 in the table, the corresponding $P$ values from eqns (43)-(45) are used.

To further verify our theory, we have selected another five fabrics with various fiber type, weave structures and Weibull strength parameters determined similarly to Ref. 16 and listed in Table 5. The yarn thickness $t_{y}$, and predictions of the fabric strength as well as its standard deviation are again calculated using the present theory.

It is shown clearly in both Tables 4 and 5 that the present predictions of both fabric strength and its variation are in reasonable agreement with the tested results. In Table 4 , our predictions are closer to the measured actual values than the predictions by Shahpurwala and Schwartz ${ }^{16}$ using either of their two models. Moreover, the general trend also shows that our predictions tend to exceed the measured strength values. We suspect that this may be due partly to the equal-load-sharing assumption adopted in the present analysis, and a less uniform load-sharing rule between the still surviving yarns will yield lower or more realistic results.

Still, there are other potential factors which may influence our predictions. One of them is the aforementioned interconnection between the gauge length and the Wcibull shape parameter $\beta_{y}$ of staple spun yarns revealed by Realff et al..$^{25}$ According to them, the failure mechanism of a yarn made of short fibers will change when the testing gauge length is below the fiber length, and at such a short gauge length, the yarn becomes unusually stronger, reflected by a reduced shape parameter $\beta_{\mathrm{y}}$ because more fibers break instead of being pulled out. If this principle is applicable here, the shape parameters determined at standard gauge length and employed in our model will underestimate the strengths of the fabrics made of short fiber yarns. However, from the data in both Tables 4 and 5 , we do not see a noticeable trend of underestimation, nor do we see a clear distinction between the predictions for fabrics made of short fiber and continuous filament yarns. This may suggest that we cannot treat the critical yarn length in eqn (11), by which our fabric strength predictions are calculated, as the equivalent gauge length in comparison to the actual testing gauge length on a tensile tester. This failure mechanism change observed on a strength tester may not dictate the yarn failure process in the fabrics used here.

Additionally, it has been reported ${ }^{30}$ that for some materials, the Weibull law of eqn (1) may overestimate the strength for shorter gauge length while underestimating the strength for longer length. It again seems not the case for the present data in the 
Table 4. Fabric strength and its SD value (in parentheses) tested and predicted (N/yarn)

\begin{tabular}{|c|c|c|c|c|}
\hline & Uncorrected & Corrected & Present model & Tested \\
\hline \multicolumn{5}{|c|}{ 1. Cotton plain } \\
\hline Warp & $3 \cdot 86(0 \cdot 12)$ & $3.93(0 \cdot 11)$ & $6 \cdot 12(0 \cdot 17)$ & $4.97(0 \cdot 17)$ \\
\hline Weft & $6.06(0.29)$ & $6 \cdot 30(0 \cdot 26)$ & $8.56(0.39)$ & $8.88(0.45)$ \\
\hline \multicolumn{5}{|c|}{ 2. Cotton twill $3 / 1$} \\
\hline Warp & $4 \cdot 70(0 \cdot 19)$ & $4 \cdot 82(0 \cdot 18)$ & $9 \cdot 14(0 \cdot 36)$ & $7 \cdot 10(0 \cdot 18)$ \\
\hline Weft & $5 \cdot 00(0 \cdot 24)$ & $5 \cdot 19(0.22)$ & $7 \cdot 22(0 \cdot 34)$ & $6 \cdot 44(0 \cdot 36)$ \\
\hline \multicolumn{5}{|c|}{ 3. Cotton satin $4 / 1$} \\
\hline Warp & $3 \cdot 84(0 \cdot 13)$ & $3.92(0 \cdot 12)$ & $5 \cdot 72(0 \cdot 18)$ & $5.09(0.20)$ \\
\hline Weft & $4 \cdot 71(0 \cdot 21)$ & $4 \cdot 88(0 \cdot 19)$ & $6 \cdot 47(0 \cdot 29)$ & $5 \cdot 75(0 \cdot 21)$ \\
\hline \multicolumn{5}{|c|}{ 4. Polyester plain } \\
\hline Warp & $1.43(0.03)$ & $1.46(0 \cdot 03)$ & $1.80(0.04)$ & $1.78(0.07)$ \\
\hline Weft & $4 \cdot 15(0 \cdot 17)$ & $4 \cdot 27(0 \cdot 15)$ & $5 \cdot 87(0 \cdot 21)$ & $4 \cdot 91(0 \cdot 34)$ \\
\hline
\end{tabular}

two tables. So our results may in fact confirm the validity of using the Weibull function to specify the yarn strength.

Another potential factor which may influence our prediction is the effect of fabric weave structures. Yet, by comparing fabrics of different structures in Tables 4 and 5, we fail to see a pattern of error associated with the weave types. This seems to indicate that the pressure and weave type relationships developed in eqns (43)-(45) work reasonably well in our case.

Table 5. Results for a new set of fabrics

\begin{tabular}{|c|c|c|c|c|c|}
\hline & \multicolumn{5}{|c|}{ Fabric $^{a}$} \\
\hline & $\begin{array}{c}1, \quad 100 \% \text { PE } \\
\text { plain }\end{array}$ & $\begin{array}{c}100 \% \text { acetate } \\
\text { plain }\end{array}$ & $\begin{array}{l}100 \% \text { acetate } \\
\text { satin }\end{array}$ & $\begin{array}{l}100 \% \text { cotton-S } 5 \\
\text { plain }\end{array}$ & $\begin{array}{l}100 \% \text { cotton-S } \\
\text { plain }\end{array}$ \\
\hline & \multicolumn{5}{|c|}{ Thread count, $n_{\mathrm{y}}$ (threads/mm) } \\
\hline Warp & 3.94 & $3 \cdot 78$ & $4 \cdot 41$ & $2 \cdot 68$ & $2 \cdot 68$ \\
\hline \multirow[t]{2}{*}{ Weft } & $3 \cdot 94$ & $2 \cdot 21$ & $2 \cdot 68$ & $2 \cdot 21$ & $2 \cdot 21$ \\
\hline & \multicolumn{5}{|c|}{ Yarn number (tex) } \\
\hline Warp & $3 \cdot 28$ & $3 \cdot 89$ & 3.99 & $39 \cdot 63$ & $36 \cdot 91$ \\
\hline \multirow[t]{2}{*}{ Weft } & 4.92 & $32 \cdot 27$ & $31 \cdot 41$ & $41 \cdot 35$ & $44 \cdot 07$ \\
\hline & \multicolumn{5}{|c|}{ Yarn thickness, $t_{\mathrm{y}}\left(10^{-2} \mathrm{~mm}\right)$} \\
\hline Warp & $2 \cdot 89$ & $3 \cdot 23$ & $3 \cdot 28$ & $9 \cdot 60$ & $9 \cdot 26$ \\
\hline \multirow[t]{2}{*}{ Weft } & $3 \cdot 54$ & $9 \cdot 33$ & $9 \cdot 21$ & $9 \cdot 80$ & $10 \cdot 12$ \\
\hline & \multicolumn{5}{|c|}{ Yarn shape parameter, $\beta_{\mathrm{y}}$} \\
\hline Warp & $20 \cdot 33$ & $45 \cdot 78$ & $12 \cdot 75$ & $11 \cdot 54$ & $14 \cdot 57$ \\
\hline \multirow[t]{2}{*}{ Weft } & $31 \cdot 53$ & $14 \cdot 50$ & $12 \cdot 84$ & $37 \cdot 77$ & $19 \cdot 54$ \\
\hline & \multicolumn{5}{|c|}{ Yarn scale parameter, $\alpha_{\mathrm{y}}\left(N^{\left.-\beta_{\mathrm{y}} / \mathrm{mm}\right)}\right.$} \\
\hline Warp & $7 \cdot 29 \times 10^{-15}$ & $1.44 \times 10^{-11}$ & $1.24 \times 10^{-5}$ & $1.82 \times 10^{-9}$ & $5.42 \times 10^{-11}$ \\
\hline \multirow[t]{2}{*}{ Weft } & $2.63 \times 10^{-21}$ & $5.68 \times 10^{-10}$ & $3.63 \times 10^{-9}$ & $4.11 \times 10^{-21}$ & $1.05 \times 10^{-13}$ \\
\hline & \multicolumn{5}{|c|}{ Yarn strength (SD) $(N /$ thread $)$, tested } \\
\hline Warp & $3 \cdot 79(0 \cdot 25)$ & $1.42(0.03)$ & $1 \cdot 54(0 \cdot 15)$ & $3 \cdot 90(0 \cdot 57)$ & $3 \cdot 67(0 \cdot 41)$ \\
\hline \multirow[t]{2}{*}{ Weft } & $3 \cdot 76(0 \cdot 13)$ & $2.93(0 \cdot 24)$ & $2.92(0 \cdot 26)$ & $3.03(0 \cdot 10)$ & $3 \cdot 50(0 \cdot 24)$ \\
\hline & \multicolumn{5}{|c|}{ Fabric strength $(\mathrm{SD})(N /$ thread $)$, tested } \\
\hline Warp & $4.40(0 \cdot 06)$ & $2.09(0.02)$ & $1 \cdot 84(0 \cdot 03)$ & $3.06(0 \cdot 19)$ & $3 \cdot 14(0 \cdot 14)$ \\
\hline \multirow[t]{2}{*}{ Weft } & $3 \cdot 78(0 \cdot 12)$ & $3 \cdot 87(0 \cdot 13)$ & $4 \cdot 04(0 \cdot 05)$ & $2 \cdot 84(0 \cdot 10)$ & $3 \cdot 64(0 \cdot 10)$ \\
\hline & \multicolumn{5}{|c|}{ Fabric strength (SD) $(N /$ thread $)$, predicted } \\
\hline Warp & $3.92(0.09)$ & $1.46(0.02)$ & $1.69(0.05)$ & $3 \cdot 88(0 \cdot 14)$ & $3 \cdot 69(0 \cdot 11)$ \\
\hline Weft & $3 \cdot 82(0 \cdot 07)$ & $3 \cdot 18(0 \cdot 11)$ & $3 \cdot 17(0 \cdot 11)$ & $2.99(0.06)$ & $3.59(0 \cdot 11)$ \\
\hline
\end{tabular}

\footnotetext{
${ }^{a} \mathrm{~F}$, filament yarn; $\mathrm{S}$, staple yarn.
} 


\section{CONCLUSIONS}

A new approach to calculate the critical yarn length for the fabric model as chain of yarn sub-bundle is proposed in this study. A woven fabric is shown to be enhanced by the yarn-yarn interactions occurring at the crossing points in a tensioned woven fabric. The yarn-yarn interactions are proven to consist of two forms; one is of adhesive nature and is independent of the pressure at the crossing point; and the other is frictional and is directly related to the normal pressure which can be generated from tensions applied to the fabric. Yet the adhesive force is proved to be generally negligible. The yarn-yarn interactions dictate the critical yarn length based on which the actual fabric strengths at uniaxial and biaxial extension cases can be calculated. The present predictions of the uniaxial loading case are shown in this study to be closer to the experimental results than those predicted by Shahpurwala and Schwartz. ${ }^{16}$ The yarn cross-section and surface property, the yarn strength, fiber volume fractions of the fabric and the yarns, and the external loads exerted on the fabric are demonstrated to be the important variables determining the fabric tensile strength.

Additionally, a more realistic stress/strain relationship for a woven fabric has been obtained in the present work by including the effect of the between-yarn strength variation which leads to a gradual breakage of yarns in the fabric under external tension.

It is also demonstrated in this study that the Weibull function is a good approximation to yarn tensile strength when used to predict fabric strength; and the yarn failure mechanism in a fabric during fabric extension is likely to be different from the yarn failure behavior observed on a strength tester using a correspondingly adjusted gauge length.

\section{ACKNOWLEDGEMENT}

The author wishes to thank the reviewer(s) for the helpful comments, especially for pointing out an error in eqn (34) in the original manuscript.

\section{REFERENCES}

1. Peirce, F. T., The geometry of cloth structure. J. Textile Inst., 28 (1937) T45.

2. Painter, E. V., Mechanics of elastic performance of textile materials: Part VIII: Graphical analysis of fabric geometry. Textile Res. J., 22 (1952) 153.

3. Love, L., Graphical relationships in cloth geometry for plain, twill and sateen weaves. Textile Res. J., 24 (1954) 1073.

4. Hearle, J. W. S., Grosberg, P. \& Backer, S., Structural Mechanics of Yarns and Fabrics, Vol. 1. WileyInterscience, New York, 1969.
5. Taylor H. M., Tensile and tearing strength of cotton cloths. J. Textile Inst., 50 (1959) T161.

6. Postle, R., Carnaby, G. A. \& de Jong, S., The Mechanics of Wool Structure. Ellis Horwood, UK, 1988, p. 207.

7. Scelzo, W. A., Backer, S. \& Boyce, M. C., Mechanistic role of yarn and fabric structure in determining tear resistance of woven cloth: 1 . Understanding tongue tear. Textile Res. J., 64 (1994) 291.

8. Scelzo, W. A., Backer, S. \& Boyce, M. C., Mechanistic role of yarn and fabric structure in determining tear resistance of woven cloth: 2. Modeling tongue tear. Textile Res. J., 64 (1994) 321.

9. Kilby, W. F., Planar stress-strain relationship in woven fabrics. I. Textile Inst., 54 (1963) T9.

10. Warren, W. E., The elastic properties of woven polymeric fabric. Polym. Eng. Sci., 30 (1990) 1309.

11. Chou, T. W., Microstructural Design of Fiber Composites. Cambridge University Press, Cambridge, 1992. p. 285.

12. Ko, F. K. \& Pastore, C. M., Structure and properties of an integrated 3D fabric for structural composites. In Recent Advances in Composites in the United States and Japan, ASTM STP 864, ed. J. R. Vinson \& M. Taya. ASTM, Philadelphia, PA, 1985, p. 428.

13. Pastore, C. M. \& Gowayed, Y. A., A self-consistent fabric geometry model: Modification and application of a fabric geometry model to predict the elastic properties of textile composites. J. Comp. Technol. Res., JC-TRER, 16 (1994) 32.

14. Hearle, J. W. S. \& Du, G. W., Forming rigid fiber assemblies: The interaction of textile technology and composites engineering. J. Textile Inst., 81 (1990) 360.

15. Li, W., Hammad, M. \& El-Shiekh, A., Forming rigid fiber assemblies: The interaction of textile technology and composites engineering. J. Textile Inst., 81 (1990) 360 .

16. Shahpurwala, A. A. \& Schwartz, P., Modeling woven fabric tensile strength using statistical bundle theory. Textile Res. J., 59 (1989) 26.

17. Daniels, H. E., The statistical theory of the strength of bundles of threads. Proc. R. Soc. Lond., $\mathbf{1 8 3}$ (1945) 405.

18. Boyce, M. C., Palmer, M. L., Seo, M. H., Schwartz, P. \& Backer, S., A model of the tensile failure process in woven fabrics. I. Appl. Polym. Sci: Appl. Polym. Symp., 47 (1992) 123.

19. Seo, M., Palmer, M. L., Pan, N., Boyce, M., Schwartz, P. \& Backer, S., On the mechanical properties of fabric woven from yarns produced by different spinning technology: Yarn failure in woven fabric. Textile Res. J., 63 (1993) 123.

20. Clulow, E. E. \& Taylor, H. M., An experimental and theoretical investigation of biaxial stress-strain relations in a plain-weave cloth. J. Textile Inst., 54 (1963) T323.

21. Freeston, W. D., Platt, M. M. \& Schoppee, M. M., Mechanics of elastic performance of textile materials: Part XVIII. Stress-strain response of fabrics under two-dimensional loading. Textile Res. I., 37 (1967) 948.

22. Reichardt, C. H., Woo, H. K. \& Montgomery, D. J., A two-dimensional load-extension tester for woven fabrics. Textile Res. J., 23 (1953) 424.

23. Skelton, J. \& Freeston, W. D., Mechanics of elastic performance of textile materials: Part XIX. The shear behavior of fabric under biaxial loads. Textile Res. J., 41 (1971) 871.

24. Pan, N. \& Yoon, M. Y., Behavior of yarn pullout from woven fabrics: Theoretical and experimental. Textile Res. J., 63 (1993) 629. 
25. Realff, M. L., Seo, M., Boyce, M., Schwartz, P. \& Backer, S., Mechanical properties of fabric woven from yarns produced by different spinning technology: Yarn failure as a function of gauge length. Textile Res. J., 61 (1991) 517.

26. Henstenburg, R. B. \& Phoenix, S. L., Interfacial shear strength studies using the single-filament-composite test. Part II: A probability model and Monte Carlo simulation. Polym. Comp., 10 (1989) 389.

27. Pan, N., A detailed examination of the translation efficiency of fiber strength into composite strength. $J$. Reinf. Plast. Comp. (in press).

28. Hatch, K. L., Textile Science. West Publishing, St Paul, MN, 1993, p. 126.

29. Smith, R. L. \& McCartney, L. N., Statistical theory of the strength of fiber bundles. J. Appl. Mech., 105 (1983) 601.

30. Watson, A. S. \& Smith, R. L., An examination of statistical theories for fibrous materials in the light of experimental data. J. Mater. Sci., 20 (1985) 8260. 